\title{
Fundamental understanding and practical challenges of anionic redox activity in Li-ion batteries
}

\author{
Gaurav Assat ${ }^{1,2,3}$ and Jean-Marie Tarascon*,1,2,3 \\ ${ }^{1}$ Collège de France, Chimie du Solide et de l'Energie - UMR CNRS 8260, 11 Place Marcelin \\ Berthelot, 75005 Paris, France \\ ${ }^{2}$ Réseau sur le Stockage Electrochimique de l'Energie (RS2E) - FR CNRS 3459, 80039 Amiens \\ Cedex, France \\ ${ }^{3}$ Sorbonne Universités, UPMC Université Paris 06, 4 Place Jussieu, 75005 Paris, France \\ *Corresponding author: J.-M. Tarascon: jean-marie.tarascon@college-de-france.fr
}

\begin{abstract}
Our increasing dependence on lithium-ion batteries for energy storage applications calls for continual performance improvements of their positive electrodes, which have so far relied solely on cationic redox of transition-metal ions for driving the electrochemical reactions. Great hope has recently been placed on the emergence of anionic redox - a transformational approach for designing positive electrodes as it leads to a near-doubling of capacity - hence generating much research interest in recent years. However, questions have been raised on the fundamental origins of anionic redox and whether its full potential can be realised in applications. In this Review, we discuss the underlying science that triggers a reversible and stable anionic redox activity. Furthermore, we highlight its practical limitations and outline possible approaches for improving such materials and designing novel ones. We also summarize their chances for market implementation in face of the competing nickel-based layered cathodes that are prevalent today.
\end{abstract}


Today's society relies on electrochemical energy storage, mainly rechargeable Li-ion batteries, to power portable electronics and electric vehicles. With the ongoing technological revolution associated with electric mobility, renewable energy integration, and connected objects, our dependence on batteries will become greater than ever. As the global demand for batteries soars, special focus remains on the popular Li-ion technology that surpasses its predecessors (leadacid, nickel-cadmium, and nickel-metal hydride) in terms of energy density (Wh $\mathrm{L}^{-1}$ ) and lifetime (years). ${ }^{1}$ The expectations are high as costs of Li-ion battery packs are projected to drop below 100 $€ \mathrm{kWh}^{-1}$ by $2020 .^{2}$

This puts pressure on the Li-ion technology to preserve its supremacy by continually improving in energy density and sustainability, bearing in mind that today's cathodes are based on cobalt - a chemical element with geopolitical and ethical concerns. ${ }^{3}$ Towards these goals, the lights are back to green with the recent discovery of anionic redox chemistry ${ }^{4-6}$, which enables a nearly doubled energy storage via electrochemical activity of ligands in Li-rich Mn-based layered oxides, e.g. $\mathrm{Li}_{1.2} \mathrm{Ni}_{0.13} \mathrm{Mn}_{0.54} \mathrm{Co}_{0.13} \mathrm{O}_{2}$ (Li-rich $\mathrm{NMC}$ ) and $\mathrm{Li}_{1.2} \mathrm{Ni}_{0.2} \mathrm{Mn}_{0.6} \mathrm{O}_{2}$, which serve as cobalt-lean alternatives for replacing today's $\mathrm{LiCoO}_{2}$ and $\mathrm{LiNi}_{1 / 3} \mathrm{Mn}_{1 / 3} \mathrm{Co}_{1 / 3} \mathrm{O}_{2}(\mathrm{NMC} 111)$ cathodes (Figure 1). ${ }^{7-12}$ The transformational anionic redox mechanism has thus emerged as a new paradigm for designing novel cathodes for high energy Li-ion batteries. ${ }^{13-15}$

As with every discovery, anionic redox was also followed by bullish performance expectations together with a blooming research activity aiming to fully understand the underlying science. Several years have passed, numerous papers have been published, and intense industrial efforts have been deployed, so that time has come to assess whether this new paradigm will ever enable the next generation of high performance Li-ion batteries. This is what this review will aim to answer. It will be structured as follows. The history of anionic redox will first be revisited, followed by a comprehensive pedagogical description of the underlying science. Then after addressing the chemical and structural principles to design new materials, the practical roadblocks of anionic- 
redox-based Li-rich materials will be highlighted. Lastly, the merits of such Li-rich cathodes will be compared against NMCs in terms of real-world applications.

\section{Emergence of anionic redox chemistry in electrode materials}

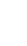

lithium (de)insertion process involving cationic redox of transition-metal ions. ${ }^{16}$ In the 1970 's, lithium-free $3 d$ transition-metal chalcogenides $\left(\mathrm{TiS}_{2}, \mathrm{MoS}_{2}, \ldots\right)$ were first identified as lithium insertion hosts for developing Li-metal batteries. ${ }^{17}$ To circumvent the safety risks concerning dendritic lithium growth at the metallic-lithium negative electrode of these batteries, the concept of Li-ion technology was proposed in the $1980 \mathrm{~s}^{18}$ with its commercialization occurring in $1991 .{ }^{19}$ This breakthrough involved the simultaneous replacement of lithium metal by carbonaceous materials at the negative electrode, and of lithium-free insertion hosts by lithium-based layered-oxide insertion compounds at the positive electrode that offered an increased cell potential due to higher electronegativity of oxygen than sulfur.

Owing to the success of Li-ion technology employing layered oxide cathodes, the original sulfide electrodes fell into oblivion despite notable scientific advances extremely relevant to the present review, especially in laying out the history of anionic redox (Box 1). Among them are the early pioneering works by Rouxel et al. ${ }^{20,21}$ on ligand-hole chemistry in sulfides, e.g. $\mathrm{TiS}_{3}$ or $\mathrm{Ti}^{4+} \mathrm{S}^{2-}\left(\mathrm{S}_{2}\right)^{2-}, \mathrm{FeS}_{2}$ or $\mathrm{Fe}^{2+}\left(\mathrm{S}_{2}\right)^{2-}$, which show the possibility of sulfur ligands to exist in a more oxidized state than $\mathrm{S}^{2-}$ by virtue of the relative positioning of metal $d$ and ligand $s p$ bands (Box 1). Besides chalcogenides, the exacerbated capacity shown by highly covalent transition-metal pnictide negative electrodes $\left(\mathrm{Li}_{x} \mathrm{MPn}_{4}, \mathrm{M}=\mathrm{Ti}, \mathrm{V}, \mathrm{Mn}\right.$ etc. and $\mathrm{Pn}=\mathrm{N}, \mathrm{P}, \mathrm{As}$, etc.) was also explained via anionic redox activity of the $\left(\mathrm{Pn}_{4}\right)^{n-}$ units. $^{22}$

Since oxides are less covalent than sulfides, anionic redox in oxides was not envisioned initially. The successful preparation of fully delithiated ' $\mathrm{Li}_{0} \mathrm{CoO}_{2}$ ', back in $1996^{23}$, was therefore 
quite puzzling as Co was not fully oxidized to $4+$, as deduced by magnetic measurements. ${ }^{24}$ Furthermore, based on the slight shortening of $\mathrm{O}-\mathrm{O}$ interplanar distances deduced by synchrotron diffraction, the participation of oxygen in the redox reaction at high potential of $\mathrm{Li}_{x} \mathrm{CoO}_{2}$ was proposed back in $1999 .^{25}$ This suggestion was supported by early theoretical papers ${ }^{26,27}$ that predicted the feasibility to design $\mathrm{LiAl}_{1-y} \mathrm{Co}_{y} \mathrm{O}_{2}$ wherein oxygen, rather than transition-metals, functions as the electron donor upon Li removal at high potential. Moreover a crude extrapolation of such calculations to the insulating $\mathrm{LiAlO}_{2}$ phase shows, as expected for a chemist, that the extracted electron will have to come from the oxygen $2 p$ orbitals since $\mathrm{Al}^{3+}$ cannot be oxidized.

For whatever reasons, such an illicit redox participation of oxygen in $\mathrm{Li}_{x} \mathrm{CoO}_{2}$ at high potential remained overlooked by the battery community for the next decade, despite being supported by additional experimental papers looking at the oxygen electronic structure using X-ray absorption spectroscopy (XAS) and X-ray photoelectron spectroscopy (XPS). ${ }^{28,29}$ This neglect was surprising, since ligand-hole chemistry was well-accepted for high-temperature superconducting cuprates $^{30}$ and is still heavily studied in the rare-earth nickelates ${ }^{31,32}$, also called "negative chargetransfer" materials. The next example, as intriguing as the electrochemical preparation of $\mathrm{Li}_{0} \mathrm{CoO}_{2}$, came a few years later with the detection of electrochemical activity in $\mathrm{Li}_{2} \mathrm{MnO}_{3}$ allegedly involving anionic redox since the oxidation of octahedral $\mathrm{Mn}^{4+}$ ions in oxides is believed to be impossible. ${ }^{33,34} \mathrm{Li}_{2} \mathrm{MnO}_{3}$, also expressed as $\mathrm{Li}\left[\mathrm{Li}_{1 / 3} \mathrm{Mn}_{2 / 3}\right] \mathrm{O}_{2}$, is made of $\mathrm{Li}$ layers sandwiched between $\mathrm{MO}_{2}\left(\mathrm{M}=\mathrm{Li}_{1 / 3} \mathrm{Mn}_{2 / 3}\right)$ layers wherein one-third of $\mathrm{Mn}$ is replaced by $\mathrm{Li}$ in a specific honeycomb-like arrangement ${ }^{4}$, hence leading to compounds with excess $\mathrm{Li}$ that are termed as Lirich layered oxides. Identifying the reaction mechanism of $\mathrm{Li}_{2} \mathrm{MnO}_{3}$ was complicated by the need to go to high potential $(>4.5 \mathrm{~V})$ to trigger electrochemical activity, hence favouring electrolyte decomposition which was shown to occur in parallel with some irreversible loss of lattice oxygen. $^{34,35}$

Despite a limited performance and a complicated mechanism, $\mathrm{Li}_{2} \mathrm{MnO}_{3}$ had the merit to 
allow various chemical substitutions aiming towards enhancing its performance. This saga, which began with $\mathrm{Ni}^{2+}$ substitution leading to $\mathrm{Li}\left[\mathrm{Li}_{(1 / 3-2 x / 3)} \mathrm{Ni}_{x} \mathrm{Mn}_{(2 / 3-x / 3)}\right] \mathrm{O}_{2}$ phases that demonstrated reversible capacities $>230 \mathrm{mAh} \mathrm{g}^{-1}$ when cycled to $4.8 \mathrm{~V}^{36}$, later bloomed with the synthesis of numerous solid-solutions of $x \mathrm{LiMO}_{2} \cdot(1-x) \mathrm{Li}\left[\mathrm{Li}_{1 / 3} \mathrm{Mn}_{2 / 3}\right] \mathrm{O}_{2}$ having $\mathrm{M}=\mathrm{Ni}, \mathrm{Mn}, \mathrm{Co}, \mathrm{Cr}$, Fe, etc. Standing out among these were the high capacity $\left(>250 \mathrm{mAh} \mathrm{g}{ }^{-1}\right) \mathrm{Li}\left[\mathrm{Li}_{0.2} \mathrm{Ni}_{0.13} \mathrm{Mn}_{0.54} \mathrm{Co}_{0.13}\right] \mathrm{O}_{2} \mathrm{Li}_{-}$ rich NMC layered oxides cathodes, ${ }^{37}$ which show a peculiar two-step charge profile followed by a sloped S-shaped discharge curve (Figure 1d) in which the cationic redox activity could only account for around half of the measured discharge capacity. Researchers thus debated upon several possibilities, such as transition-metal over-oxidation ${ }^{33}$, irreversible oxygen loss with surface densification ${ }^{35,38,39}, \mathrm{Li}^{+} / \mathrm{H}^{+}$exchange ${ }^{34,40}, \mathrm{Li}_{2} \mathrm{O}$ removal with ' $\mathrm{MnO}_{2}$-like' activation ${ }^{37,41}$, oxygen release/re-accommodation ${ }^{42}$, oxygen redox at the interphase ${ }^{43}$, and reversible redox of bulk lattice oxygen $^{4,6,44,45}$. All controversies have converged and it is now well accepted, based on complementary experimental ${ }^{46-50}$ and theoretical ${ }^{51-53}$ works, that the extraordinary capacity offered by Li-rich NMC is due to the cumulative contribution of both cationic and anionic reversible redox processes in the bulk.

Such a unified view was made possible by first designing model Li-rich compounds, namely lithium ruthenates $\left(\mathrm{Li}_{2} \mathrm{Ru}_{1-y} \mathrm{Sn}_{y} \mathrm{O}_{3}\right)$ that are structurally and electrochemically similar to the Li-rich NMC phases ${ }^{5}$, while having a simpler redox chemistry since $\mathrm{Ru}$ is the only redox-active cation in comparison to Li-rich NMCs that contain three different redox-active cations ( $\mathrm{Ni}, \mathrm{Co}$ and $\mathrm{Mn}$ ). For these model electrodes, electron paramagnetic resonance $(E P R)^{54}$, which detects single-spin or radical species, was used to unambiguously detect 'peroxo-like' species in the charged materials which was further confirmed using $\mathrm{O} 1 s \mathrm{XPS}^{48}$. This finding was firmly established with the visualization of $\mathrm{O}-\mathrm{O}$ peroxo-like dimers in the model $\mathrm{Li}$-rich $\mathrm{Li}_{2} \mathrm{IrO}_{3}$ phases via transmission electron microscopy (TEM) and neutron diffraction. ${ }^{55}$ These experimental proofs were further complemented by density functional theory (DFT) calculations ${ }^{51-53,56}$, and using theoretical tools 
such as the projected density of states for identifying the cation/anion band positions, the Fukui function to identify redox centers ${ }^{55}$, and the crystal orbital overlap population (COOP) plots to show $\mathrm{O}-\mathrm{O}$ bond formation ${ }^{52}$.

The demonstration of anionic redox in $4 d$ and $5 d$ based model Li-rich cathodes served as a platform to assess if this scenario could be naturally extended to explain the complicated charge compensation mechanism in Li-rich NMC. Providing a straight answer was not easy as direct TEM visualization of the oxygen network, like neatly done in $\mathrm{Li}_{2} \mathrm{IrO}_{3}$ to spot $\mathrm{O}-\mathrm{O}$ dimers, has not been possible in Li-rich NMC due to the absence of a clear structure projection. On the other hand, EPR could not be used due to signal interferences between the transition metals and oxygen. Lastly, XPS on Li-rich NMC proved the appearance of oxidized lattice oxygen, but doubts still remained because of the limited probe-depth of in-house XPS. ${ }^{48}$ This is no longer the case owing to recent measurements with hard-XPS (or HAXPES) ${ }^{49}$ having higher probe-depths, and bulk-sensitive O Kedge XAS measurements ${ }^{46,47,50}$, which showed that the anionic redox activity in Li-rich NMC is truly a bulk process.

Altogether the aforementioned contributions have commenced a new era in battery research which views anionic redox as a transformational change for creating advanced electrode materials, and several novel ones have already been found. As the field expands, there is a need for returning to fundamentals and provide a theoretical rationale for the underlying science, as addressed next.

\section{The science underlying the anionic redox process}

In the band structure of insertion compounds, the Fermi level $\left(E_{F}\right)$ can be related to their electrochemical redox potential such that holes above $E_{F}$ and electrons below $E_{F}$ form a redox couple. ${ }^{16}$ The band structure of lithium-based transition-metal oxides simply considers orbitaloverlaps between the transition-metal $d$ orbitals and the oxygen $p$ orbitals resulting in bonding (M- 
$\mathrm{O}$ ) and antibonding $(\mathrm{M}-\mathrm{O})^{*}$ bands having, respectively, strong ligand and metal characters (Figure 2a-c). The energy difference between $(\mathrm{M}-\mathrm{O})$ and $(\mathrm{M}-\mathrm{O})^{*}$, also called the charge transfer term $\Delta$, depends on the electronegativity difference $\Delta \chi$ between $\mathrm{M}$ and $\mathrm{O} . \Delta$ reflects the iono-covalent character of the $\mathrm{M}-\mathrm{O}$ bonds, e.g. $\Delta$ decreases (lower ionicity) by replacing $\mathrm{O}$ with less electronegative $\mathrm{S}$, and this trend continues from $\mathrm{S}$ towards Te. For classical cathodes, their redox process was so far believed to involve solely the (M-O)* band (Figure 2c) having strong metal character, hence the term cationic redox.

Recently theorists recognized the occurrence of non-bonding oxygen states in the band structure of Li-rich materials through simple Lewis descriptions. ${ }^{52}$ The Lewis configuration of $\mathrm{O}^{2-}$ enlists one $2 s$ and three $2 p$ doublets, the former being redox-inactive by lying deep in energy. In contrast, the higher energy $\mathrm{O} 2 p$ doublets participate in $\mathrm{M}-\mathrm{O}$ bond formation with the degree of involvement being structure dependent. All three $2 p$ orbitals engage in $\mathrm{M}-\mathrm{O}$ bond formation in classical layered $\mathrm{LiMO}_{2}(\mathrm{O} / \mathrm{M}=2$, Figure 2d), unlike in structures having higher O/M ratios, e.g. lithium-rich $\mathrm{Li}_{2} \mathrm{MO}_{3}$ (Figure 2e) wherein one of the $\mathrm{O} 2 p$ orbitals, the one pointing towards $\mathrm{Li}$ in the $\mathrm{Li}_{1 / 3} \mathrm{M}_{2 / 3} \mathrm{O}_{2}$ layer, is weakly bonded owing to its large energy difference from the Li $2 s$ orbital. Hence, it behaves like an $\mathrm{O}$ non-bonding state (also sometimes called 'orphaned or unhybridized $\mathrm{O}$ $2 p$ state', or 'O lone-pair', or ' $\mathrm{Li}-\mathrm{O}-\mathrm{Li}$ configuration', or ' $b_{1}$ * state in $\mathrm{C}_{2 v}$ point-group symmetry', leading to unnecessary confusion arising from semantics) $)^{46,51,53,57}$ and is located above the stabilized (M-O) bonding band (Figure 2c).

Why is this $\mathrm{O}$ non-bonding state electrochemically so interesting? Simply because it offers, besides the usual $(\mathrm{M}-\mathrm{O})^{*}$ band, a second band for removing extra electrons and gaining capacity without the risk of structural destabilization, unlike in classical systems where extra electrons can only come from the stabilized $(\mathrm{M}-\mathrm{O})$ bonding bands once $(\mathrm{M}-\mathrm{O})^{*}$ is emptied. Triggering such a two-band redox process depends on the respective positions of $(\mathrm{M}-\mathrm{O})^{*}$ antibonding and $\mathrm{O} 2 p$ non-bonding bands. 
To better assess the band positioning, we need to first introduce herein the $d$ - $d$ Coulomb interaction term $U$, usually not explicitly sketched by battery chemists but frequently used by solidstate physicists to characterize the on-site electron repulsion within the $d$ orbitals. ${ }^{58}$ This term, which tends to favour on-site localized electrons as opposed to the kinetic energy, splits the partially filled $(\mathrm{M}-\mathrm{O})^{*}$ band, called Mott-Hubbard splitting, resulting in empty upper- and filled lowerHubbard bands (UHB and LHB respectively, Figure 2f). More quantitatively, $U$ is inversely proportional to the orbital volume and hence strongly depends on the $d$ metal involved. Therefore, it increases from left to right of the periodic table $\left(\mathrm{Ti}^{n+} \rightarrow \mathrm{Ni}^{n+}\right)$ due to orbital contraction, and it decreases from $3 d$ to $5 d$ transition-metals due to orbital expansion.

The position of LHB with respect to $\mathrm{O} 2 p$ non-bonding band thus depends on the relative values of $U$ vs. $\Delta$, giving rise of three different scenarios (Figure $2 \mathrm{f}-\mathrm{h}$ ). ${ }^{53}$ First for $U<<\Delta$, a situation that widely applies to oxides and fluorides having highly ionic (large $\Delta$ ) $\mathrm{M}-\mathrm{L}$ bonds ( $\mathrm{L}=$ ligand), electrons are exchanged from the filled LHB alike the classical one-band cationic redox scenario (Figure 2f). Turning to the opposite situation of highly correlated systems with $U>\Delta$ (Figure 2h), the one-band redox process still persists however with the electrons now directly removed from the non-bonding $\mathrm{O} 2 p$ band sitting above the filled LHB. This situation creates, owing to the high chemical hardness of localized non-bonding $\mathrm{O} 2 p$ states, highly reactive $\mathrm{O}^{n-}$ species that may de-coordinate from the metallic network via reductive elimination or by attacking the electrolyte, hence leading to partially irreversible processes as observed for $\mathrm{Li}_{2} \mathrm{MnO}_{3}{ }^{34,35}$ and related $\mathrm{Li}$-rich $\mathrm{NMCs}^{38,46,59}$, or $\mathrm{Li}_{4} \mathrm{FeTeO}_{6}{ }^{60}$ and $\mathrm{Li}_{4} \mathrm{FeSbO}_{6}{ }^{61}$ cathodes. For Li-rich NMCs, to what extent the surrounding $\mathrm{Mn}^{4+}$ cations stabilize, as recently proposed ${ }^{46}$, the $\mathrm{O}^{n-}$ species? Certainly not fully, since oxygen loss is always evidenced over the first charge of these materials. Note that this simultaneous oxygen and Li removal obviously modifies Li-rich NMCs' initial band structure, which may afterwards become more favourable for reversible anionic redox and/or the formation of $\mathrm{O}-\mathrm{O}$ dimers. Capturing such dynamic changes of the band structure requires further DFT 
development.

Lastly, the middle situation of $U / 2 \approx \Delta$ (Figure $2 \mathrm{~g}$ ) results in overlapping LHB and $\mathrm{O} 2 p$ non-bonding bands, which are simultaneously available for electrochemical activity that can occur either sequentially $\left(\mathrm{Li}_{2-x} \mathrm{RuO}_{3}\right)^{62}$ or simultaneously $\left(\mathrm{Li}_{2-x} \mathrm{IrO}_{3}\right)^{55,63}$ resulting in a doubled capacity. In this case, removal of electrons leads to a degenerated Fermi level which is unstable. To circumvent this instability, the degeneracy is lifted via either Jahn-Teller or Peierls distortions that consist of oxygen network reorganization and lowering of symmetry to shorten some $\mathrm{O}-\mathrm{O}$ distances and enable stabilizing $\mathrm{M}-\left(\mathrm{O}_{2}\right)^{n-}$ interactions. ${ }^{53}$ Such stabilization of the peroxo-like $\mathrm{O}-\mathrm{O}$ dimers through covalent interactions from the transition-metal was previously termed as reductive coupling $^{5,52}$, drawing an analogy with coordination chemistry. The above description explains the experimentally observed distortion of $\mathrm{MO}_{6}$ octahedra in $\mathrm{Li}_{2} \mathrm{IrO}_{3}{ }^{55,63}$ and $\mathrm{Li}_{2} \mathrm{RuO}_{3}{ }^{56,62}$. We thus have a unique situation in which the electrons are partially removed from the anion's non-bonding band, hence the term anionic redox. This is totally different from highly delithiated $\mathrm{LiCoO}_{2}$ where $\mathrm{O}$ appears redox-active, as deduced by Bader charge calculations ${ }^{26}, \mathrm{XAS}^{28}$, or XPS ${ }^{29}$ analyses, simply because of high covalence that imparts a significant $\mathrm{O}$ character to the redox-active $(\mathrm{M}-\mathrm{O})^{*}$ band. We hence caution against calling this situation as anionic redox since it basically remains just a oneband process offering no extra capacity.

Overall in light of these band diagrams, the situation of interest for extra capacity requires $U / 2 \approx \Delta$, thus opening the door for materials designers to play with the delicate balance between $\Delta$ and $U$ by properly choosing metal-ligand combinations. In contrast, the situation to definitely avoid is the one corresponding to irreversible $\mathrm{O}_{2}$ loss. Theorists have thoroughly explored this frontier by calculating the enthalpy of oxygen loss reaction $\left(\mathrm{Li}_{x} \mathrm{MO}_{3} \rightarrow \mathrm{Li}_{x} \mathrm{MO}_{3-\delta}+\delta / 2 \mathrm{O}_{2}\right)$ as a function of Li content for all $3 d, 4 d$, and $5 d$ metals, and have highlighted that the difficulty to avoid this prohibited situation is greater with $3 d$ metals than with $4 d$ or $5 d$ ones. ${ }^{53,64}$ Although such a theoretical conclusion is pessimistic considering raw material costs, we should not give up with $3 d$ 
metals and various engineering strategies to slow down the oxygen loss in Li-rich NMC are already being pursued. ${ }^{7,65}$

For sake of completeness, we emphasize that neither high covalence nor the presence of ligand non-bonding $p$ bands are individually sufficient conditions to ensure a reversible anionic redox. For example concerning covalence, although early XAS measurements on $\mathrm{TiS}_{2}$ showed a strong redox involvement of $\mathrm{S}^{66}$, it was simply due to the high ligand character of antibonding (MS)* states because of high covalence and it does not give extra capacity via what we herein call the anionic redox process. Likewise for non-bonding $\mathrm{O} 2 p$ states, they also exist in polyanionic compounds, such as $\mathrm{LiFePO}_{4}$ or $\mathrm{LiFeSO}_{4} \mathrm{~F}$, which solely show cationic redox because such states are too deep in energy (too far from $E_{F}$ ) to be redox-active in these ionic structures.

Although the abovementioned picture is built around oxides, it also fully rationalizes the early works on chalcogenides. For instance in the tri-sulfide $\mathrm{TiS}_{3}$, electrochemical Li uptake first accompanies the disappearance of $\mathrm{S}-\mathrm{S}$ dimers via anionic reduction of $\left(\mathrm{S}_{2}\right)^{2-}+2 \mathrm{e}^{-} \leftrightarrow 2 \mathrm{~S}^{2-}$, followed by $\mathrm{Ti}^{4+/ 3+}$ reduction, as neatly evidenced by ex situ XPS measurements. ${ }^{67}$ Put simply, $\mathrm{Ti}^{4+}$ in $\mathrm{TiS}_{3}$ cannot satisfy the $\mathrm{S}^{2-}$ state $\left(\mathrm{Ti}^{6+}\right.$ is not possible), thus triggering a structural distortion to remix the empty S $3 p$ non-bonding levels and to form $\left(\mathrm{S}_{2}\right)^{2-}$ dimers which eventually stabilize the $\operatorname{TiS}^{2-}\left(\mathrm{S}_{2}\right)^{2-}$ structure in which $\left(\mathrm{S}_{2}\right)^{2-}$ can be electrochemically reduced as shown above. Let's recall that such anion-anion interactions can go far beyond just dimers in a highly covalent lattice such as $\operatorname{IrTe}_{2}$ (or $\left.\operatorname{Ir}^{3+}\left(\mathrm{Te}^{3 / 2-}\right)_{2}\right)$, which demonstrates a Te-Te sub-lattice polymerization to form a 'polymerized $\mathrm{CdI}_{2}$-type' structure. ${ }^{20,21,68}$ Although satisfactorily describing Li-rich oxides and chalcogenides, the overall explanation relying on filled non-bonding ligand states will be continually challenged with the discovery of new materials showing unexpected features. Within this context, preliminary claims of anionic redox in Na-poor layered oxides having no non-bonding levels, although not unambiguously demonstrated yet, is already intriguing. ${ }^{69}$ Besides pinning down the anionic redox mechanism, theory has also provided guidance in the search for novel high 
capacity materials that are discussed next.

\section{Widening the spectrum of oxides showing anionic redox}

In light of the anionic redox mechanism established above, a mastering of the relative position of cationic vs. anionic levels is sorely needed for solid-state chemists to uncover novel anionic-redox materials. The preferred approach of chemical substitutions, which has already marked the successful 25 year-long journey of layered oxide cathodes from the simple $\mathrm{LiCoO}_{2}$ towards $\mathrm{LiNi}_{x} \mathrm{Mn}_{y} \mathrm{Co}_{1-x-y} \mathrm{O}_{2}(\mathrm{NMC})$ and $\mathrm{Li}\left[\mathrm{Li}_{1-x-y-z} \mathrm{Ni}_{x} \mathrm{Mn}_{y} \mathrm{Co}_{z}\right] \mathrm{O}_{2}$ (Li-rich $\mathrm{NMC}$ ) phases, could again be fruitful here. Li-rich NMCs were subjected to intense chemical manipulations aiming performance enhancement, which enlisted partial substitutions of $3 d$ metals for $\mathrm{Cr}, \mathrm{Al}$, $\mathrm{Ti}$, Mo etc., alkali $\mathrm{Li}$ for $\mathrm{Na}$ and $\mathrm{K}$, and even of $\mathrm{O}$ for $\mathrm{F}$, as comprehensively listed in other reviews. ${ }^{7-9,11}$ The quest for novel anionic redox cathodes beyond Li-rich NMC went naturally in the direction of increasing the Li-rich character (Figure 3), resulting in a wide variety of new materials with versatile compositions, crystal-structural dimensionality, and structural order/disorder, the most interesting of which are discussed herein whereas a complete listing can be found in other reviews. ${ }^{14,15,57,70,71}$

Owing to the richness of the layered rock-salt $\mathrm{Li}_{2} \mathrm{MO}_{3}$ family, great effort was initially placed in exploring elements other than $3 d$ metals such as Mn. This led to the work on $4 d$ and $5 d$ metals giving rise to ruthenates $\left(\mathrm{Li}_{2} \mathrm{RuO}_{3}\right)$ and iridates $\left(\mathrm{Li}_{2} \mathrm{IrO}_{3}\right)$ that show capacities exceeding 230 $\mathrm{mAh} \mathrm{g}^{-1}$.,55 $^{\mathrm{T}} \mathrm{Th}_{2} \mathrm{MO}_{3}$ family was further explored by designing phases of the general formula $\mathrm{Li}_{4} \mathrm{MM}^{\prime} \mathrm{O}_{6}$, within which $\mathrm{M}$ and $\mathrm{M}^{\prime}$ can be selected either from di-, tri-, tetra-, penta-, or hexavalent cations as long as their valence sum equals eight. Examples include $\mathrm{Li}_{4} \mathrm{Fe}^{2+} \mathrm{Te}^{6+} \mathrm{O}_{6}{ }^{60}$ and $\mathrm{Li}_{4} \mathrm{Fe}^{3+} \mathrm{Sb}^{5+} \mathrm{O}_{6}{ }^{61}$ that copiously release gas, and $\mathrm{Li}_{4} \mathrm{Ni}^{2+} \mathrm{Te}^{6+} \mathrm{O}_{6}{ }^{72}$ that doesn't show anionic participation, hence revealing the delicate balance between anionic redox and $\mathrm{O}_{2}$ release. A particular attention was dedicated to the model $\mathrm{Li}_{2}(\mathrm{Ru}, \mathrm{M}) \mathrm{O}_{3}$ system containing either $\left(\mathrm{Mn}^{4+}\right)^{4}$ or $d^{0}$ 
$\left(\mathrm{Ti}^{4+}, \mathrm{Zr}^{4+}\right)^{73,74}$ and $d^{10}\left(\mathrm{Sn}^{4+}\right)^{5}$ metals as substituents. Through these works and especially by comparing the Sn- and Ti-substituted phases, it could be deduced that voltage fade was exacerbated in the latter and mainly rooted in the migration of small-sized (hence more mobile) Ti ions from octahedral sites, together with their capturing in tetrahedral sites ${ }^{73}$. These works therefore provided a chemical clue to mitigate voltage fade, i.e. incorporation of large-sized cations such as $\mathrm{Sn}$, which unfortunately has been difficult so far to implement in the synthesis of Li-rich NMC.

Another interesting chemical direction, driven by the willingness to probe the effect of modifying the crystal structure on the anionic redox reactivity, involved the testing of various disordered rock-salt structures having an excess amount of $\mathrm{Li}^{14}$ Such compounds with $\mathrm{Li} / \mathrm{M}$ ratio > 1 are derived from either $\mathrm{Li}_{2} \mathrm{TiO}_{3}{ }^{75,76}, \mathrm{Li}_{3} \mathrm{NbO}_{4}{ }^{75,77}, \mathrm{Li}_{4} \mathrm{MoO}_{5}{ }^{78-80}$, or $\mathrm{Li}_{5} \mathrm{ReO}_{6}{ }^{78}$, by partially substituting the metal cations with $3 d$ metals having $d$ electrons for both weight minimization and electronic conductivity enhancement as the unsubstituted phases are highly insulating. Within this context, numerous Li-rich $\mathrm{Li}_{2-x-y} \mathrm{Nb}_{x} \mathrm{M}_{y} \mathrm{O}_{2}(\mathrm{M}=\mathrm{V}, \mathrm{Mn}, \mathrm{Fe}, \mathrm{Co}$, and $\mathrm{Ni})$ systems were tested with the most interesting results obtained with the disordered rock-salt composition of $\mathrm{Li}_{1.3} \mathrm{Nb}_{0.3} \mathrm{Mn}_{0.4} \mathrm{O}_{2}$ that reaches capacities of $300 \mathrm{mAh} \mathrm{g}^{-1}$ at $50^{\circ} \mathrm{C}^{75,77} \mathrm{Nb}^{5+}\left(d^{0}\right)$ was chosen here on the valid recognition by the authors that peroxides indeed easily form with $d^{0}$ elements $\left(\mathrm{CaO}_{2}\right)$. Although demonstrating a high capacity, application-wise, such disordered rock-salt phases show sluggish kinetics with moderate capacity retention, likely due to the total intermixing of $\mathrm{Li}, \mathrm{Nb}$, and $3 d$-metal cations, which prevents well-defined 3-D Li diffusion pathways. Similar limitations are also present for the so-called ' $\mathrm{Li}_{4} \mathrm{Mn}_{2} \mathrm{O}_{5}$ ' disordered rock-salt phase. ${ }^{81}$ Interestingly, theoretical predictions had been more optimistic about Li diffusion in Li-excess disordered phases ${ }^{82}$, hence further work is needed. The effect of two-dimensional (2-D) vs. three-dimensional (3-D) structures on the anionic redox systems was elucidated by further exploring polymorphism in $\mathrm{Li}_{2} \mathrm{IrO}_{3}$ which exhibits a layered 2-D $\alpha-\mathrm{Li}_{2} \mathrm{IrO}_{3}$ polymorph and a 3-D $\beta-\mathrm{Li}_{2} \mathrm{IrO}_{3}$ polymorph. This 3-D compound was shown to reversibly exchange $2 \mathrm{Li}$ per transition-metal atom via insertion mechanism with good 
capacity retention and high rate capability at room temperature. ${ }^{63}$ Moreover, such a large activity was shown to result from joint reversible cationic and anionic redox processes as deduced via complementary XPS, TEM, and neutron diffraction experiments supported by DFT calculations, hence ending the long-held belief that anionic redox process could solely exist in layered (2-D) materials. Unlocking this dimensionality constraint has broadened the possibilities for designing high energy-density electrodes based on anionic redox, since 3-D oxides are the largest class of existing materials.

Driven by the rationale that increasing the number of $\mathrm{O} 2 p$ non-bonding states should increase the capacity of Li-rich materials, another recently developed direction has consisted in designing materials with $\mathrm{Li} / \mathrm{M}$ or $\mathrm{O} / \mathrm{M}$ ratios greater than 2 and 3 respectively, i.e. departing from $\mathrm{Li}_{2} \mathrm{MO}_{3}$ and moving towards $\mathrm{Li}_{3} \mathrm{MO}_{4}, \mathrm{Li}_{4} \mathrm{MO}_{5}$, and so on. Note that all such compositions can be expressed as $\mathrm{Li}_{1+y} \mathrm{M}_{1-y} \mathrm{O}_{2}$ (e.g. $y=1 / 3$ for $\mathrm{Li}_{2} \mathrm{MO}_{3}$ and $y=1 / 2$ for $\mathrm{Li}_{3} \mathrm{MO}_{4}$ ) where $y$ also represents the fraction of non-bonding $\mathrm{O} 2 p$ states (e.g. 1 out of 3 in $\mathrm{Li}_{2} \mathrm{MO}_{3}$ ). Along this strategy, the most interesting behaviour was obtained via the design on a novel layered $\mathrm{Li}_{3-x} \mathrm{IrO}_{4}$ compound ${ }^{83}$ from which $2 \mathrm{Li}$ can be removed through a charge compensation process solely involving anionic oxidation to produce a $\mathrm{LiIrO}_{4}$ phase which upon further delithiation was irreversibly releasing $\mathrm{O}_{2}$. However by limiting the charge process to $x=2$, such a phase could reversibly exchange $3.5 \mathrm{e}^{-}$per transition-metal atom via intercalation mechanism with the cumulative activity of anionic $\left(\mathrm{LiIr}^{5+} \mathrm{O}_{4}\right.$ $\left.\leftrightarrow \mathrm{Li}_{3} \mathrm{Ir}^{5+} \mathrm{O}_{4}\right)$ and cationic $\left(\mathrm{Li}_{3} \mathrm{Ir}^{5+} \mathrm{O}_{4} \leftrightarrow \mathrm{Li}_{4.5} \mathrm{Ir}^{4+/ 3+} \mathrm{O}_{4}\right)$ redox processes, leading to a nearly stable reversible capacity of $356 \mathrm{mAh} \mathrm{g}^{-1}$ for at least 25 cycles, with a part of it coming at low potential. This new approach is appealing but it must be realized that pushing oxygen redox brings about complications since highly oxidized $\mathrm{MO}_{y}$ materials become increasingly unstable towards $\mathrm{O}_{2}$ release or even decomposition, as reported for $\mathrm{Li}_{3} \mathrm{RuO}_{4}$ that presents a 1-D chain structure. ${ }^{84}$ In this regard, Figure 3 demonstrates a clear trend towards lower dimensional structures as $\mathrm{Li} / \mathrm{M}$ and $\mathrm{O} / \mathrm{M}$ ratios are pushed up to increase the amount of $\mathrm{O} 2 p$ non-bonding states, ultimately leading to 0 -D 
structures like $\mathrm{Li}_{3} \mathrm{NbO}_{4}$ or $\mathrm{Li}_{4} \mathrm{MoO}_{5}$ that have isolated $\mathrm{Nb}_{4} \mathrm{O}_{16}$ clusters or isolated $\mathrm{Mo}_{2} \mathrm{O}_{10}$ units respectively, while the extreme cases of $\mathrm{Li}_{5} \mathrm{ReO}_{6}$ and $\mathrm{Li}_{7} \mathrm{RuO}_{6}$ show isolated $\mathrm{MO}_{6}$ octahedra. As such 0-D structures are not suitable for conductive and stable lithium insertion cathodes, the $\mathrm{O} / \mathrm{M}$ ratio cannot be arbitrarily pushed and there is a need to find a trade-off between extra-capacity and structural stability against $\mathrm{O}_{2}$ release. This is a difficult task as we want to use light and practical $3 d$ metals which is not supported by theoretical predictions. ${ }^{53,64}$ Alternatively, the O/M ratio's upper limits may be breached by moving beyond insertion hosts to instead design fundamentally different conversion-type electrodes having small TM amounts. Along that line, worth mentioning is the emergence of nanocomposite electrodes, named ' $\mathrm{Co}$-doped $\mathrm{Li}_{2} \mathrm{O}$ ' ${ }^{85,86}$. They function mainly via solid-phase anionic redox, i.e. $\mathrm{Li}_{2} \mathrm{O}(\mathrm{s}) \leftrightarrow \mathrm{Li}_{2} \mathrm{O}_{2}$ (s) $\leftrightarrow \mathrm{LiO}_{2}$ (s), while avoiding $\mathrm{O}_{2}$ (g) release thanks to an overcharge-preventing shuttle. Although attractive in terms of specific energy, further studies are awaited to evaluate their real-world potential.

This materials discovery pathway (Figure 3), together with the adoption of new characterization techniques by the battery community to detect specifically the electrochemical activity of oxygen (Box 2), has enabled the exploration of many facets that govern the anionic redox process, such as disorder, dimensionality, $\mathrm{O} / \mathrm{M}$ ratio, and stability against $\mathrm{O}_{2}$ release. Despite such a prolific chemistry, the most interesting materials so far rely on $4 d$ or $5 d$ rather than $3 d$ metals, thereby resulting in model materials for fundamental studies rather than practical compounds. Nevertheless, these model compounds have enabled the establishment of a sound scientific platform for rationalizing the design of future anionic-redox-based cathodes. Moreover, they have helped in unravelling the origins of practical roadblocks in Li-rich cathodes, as we touch upon next.

\section{Practicability of anionic redox in Li-rich cathodes}

After witnessing how the promise of higher capacity from anionic redox triggered the 
design of novel cathodes with compositional and structural versatility, we next evaluate their practicability concerning real-world applications where higher capacity is just one of the several stringent requirements. Besides showing higher capacity, a novel cathode must also outperform the existing ones in terms of rate capability, energy efficiency, and cycling stability, while staying competitive in cost and safety. Worth mentioning is the need for updated electrochemical testing protocols beyond classical cathodes (Box 3), focusing specifically on the unique electrochemical properties that concern anionic redox compounds.

Let's start with the popular Li-rich NMC, which still awaits commercial success despite years of academic and industrial efforts, and for which we can spot practical issues right from the first cycle. Its characteristic two-stepped first charge profile (recall Figure 1d) starts with a classical intercalation-type cationic redox step that is problem-free ${ }^{87,88}$, followed by an anionic redox plateau that terminates with irreversible gas release. ${ }^{46,59}$ Besides being an obvious source of parasitic reactions that affect cell-life, such irreversibility also calls for over-balanced (heavier) full-cells. Moreover, anionic oxidation permanently modifies the electrochemistry, inducing S-shaped charge/discharge curves. Although a large capacity is delivered via combined cationic-anionic redox activity once this sloped profile stabilizes, a substantial voltage hysteresis $(>400 \mathrm{mV})$ still remains, thus penalizing energy efficiency (Figure 4a). This hysteresis, of thermodynamic rather than kinetic origins (i.e. it does not vanish at near-zero current ${ }^{87,89}$, and also evidenced from the asymmetric charge vs. discharge $d Q / d V$ profiles), leads to path dependence that complicates stateof-charge (SoC) management. Since hysteresis decreases the energy efficiency, causing energy wastage in every cycle (presumably dissipated as heat), it becomes a cost-issue especially for largescale applications such as electric vehicles and stationary storage. ${ }^{90}$ Therefore this feature, which was also a nail in the coffin for the much-hyped conversion-based anodes, should not be ignored for Li-rich NMC.

Only a few studies have tried to understand the fundamental origins of hysteresis. 
Among them are voltage window experiments ${ }^{10,49,87}$ that showed how anionic oxidation at high potential corresponds to a reduction at substantially lower potential. Nuclear magnetic resonance (NMR) measurements ${ }^{91}$, complemented with phase-change ${ }^{89}$ and lattice-gas ${ }^{92}$ models, claimed reversible cationic migration to be correlated with voltage hysteresis. Whether such a correlation implies causation remains open for debate, especially over extended cycling. Interestingly, a recent study also pointed out the correlation between anionic redox and cation migration, claiming a coupling between the two effects. ${ }^{50}$ It must however be recalled that cationic migration is in fact a consequence of unstable structure after anionic oxidation. Along that line, combined spectroscopic (HAXPES and XAS) and electrochemical characterizations have recently demonstrated a direct association of anionic redox with voltage hysteresis (Figure 4c). ${ }^{49,93}$ It was additionally established, by linking the electrochemical impedance of Li-rich NMC to its charge-compensation mechanism, that anionic redox also suffers from sluggish kinetics at high as well as at low potentials in contrast to the fast cationic redox (Figure $4 \mathrm{c}$ ). ${ }^{49}$ As a combined consequence of voltage hysteresis and sluggish kinetics, the overall polarization in Li-rich cathodes becomes quite large. As of today, a still remaining fundamental question, which calls for theoretical developments, is to answer why anionic redox is correlated with the issues of hysteresis and sluggish kinetics. A plausible hypothesis could be the energy consuming (hence sluggish) short-range atomic movements associated to the repeated anionic-redox-driven migration of TM ions and/or to the repeated formation/breaking of $\mathrm{O}-\mathrm{O}$ dimers upon charge/discharge. Therefore, structural flexibility of the oxygen network can be envisaged as a key requirement to counter these issues.

On the experimental side, hysteresis in Li-rich NMC has not been overcome despite numerous chemical strategies attempted in literature (see other reviews ${ }^{7-9,11}$ ). Interestingly, this issue is still present but less severe $(\sim 200 \mathrm{mV})$ in $4 d$-based Li-rich material $\mathrm{Li}_{2} \mathrm{Ru}_{0.75} \mathrm{Sn}_{0.25} \mathrm{O}_{3}$ (LRSO) (Figure 4a) where hysteresis was further shown to be clearly triggered at high potential at which anionic redox occurs. ${ }^{94}$ Moreover, similar to Li-rich NMC but less severe, an asymmetry in 
$d Q / d V$ profiles exists for LRSO, and it arises from anionic redox, as visualized via operando XAS that enabled a decoupling of cationic-anionic processes in the $d Q / d V$ plot (see panel $\mathrm{g}$ in Box 2). ${ }^{62}$ Besides hysteresis, the LRSO model system also facilitated the elucidation of sluggish anionic kinetics via electroanalytical measurements (Figure 4b). ${ }^{94}$ Overall, the similar findings between Lirich NMC (3d-practical system) and LRSO (4d-based model system), regarding the detrimental role of anionic redox in triggering hysteresis and sluggish kinetics, are intriguing, especially because other anionic redox cathodes also appear to show similar or worse limitations. For example, the promising low-cost high-capacity $\mathrm{Li}_{1.2} \mathrm{Mn}_{0.4} \mathrm{Ti}_{0.4} \mathrm{O}_{2}$ rock-salt composition exhibits a huge voltage difference between charge and discharge even at $50{ }^{\circ} \mathrm{C}$ (Figure $4 \mathrm{a}$, bottom), thus reminiscent of both sluggish kinetics and hysteresis. ${ }^{75}$ The only exceptions to these limitations are the $\mathrm{Li}_{2} \mathrm{IrO}_{3}$ polymorphs as they show superimposing charge-discharge voltage curves (Figure 4a, top). ${ }^{55,63}$ Overall, depending on the chemical composition and covalence, the interplay between cationicanionic redox processes governs the practically important properties of kinetics and hysteresis in Lirich materials.

Unlike these two relatively new concepts, the early identified drawback of voltage fade in Li-rich NMC, which gradually lowers its energy output and complicates SoC management, has been extensively investigated. ${ }^{7,10}$ It is now well-established that voltage fade is promoted in Li-rich $\mathrm{NMC}$ at high potential where anions are redox-active ${ }^{49,95}$, with the same effect further validated in the LRSO model system ${ }^{94}$. Moreover, LRSO helped discovering a mitigation strategy via Snstabilized $\mathrm{Li}_{2} \mathrm{RuO}_{3}$ that shows remarkably mollified voltage fade (Figure $4 \mathrm{~d}$ ) in contrast to the Tisubstituted case. ${ }^{73}$ This was explained by the gradual capturing of small-sized Ti ions inside tetrahedral sites over long cycling. The same rationale accounts for voltage fade in Li-rich NMC, owing to the small size of Mn ions that promote a gradual 'layered to spinel' transition facilitated by oxygen loss when cycled at high potentials. ${ }^{10,88}$ Various chemical strategies, ranging from surface modification and chemical substitution to electrolyte additives, were pursued to solve 
voltage fade in Li-rich NMC, as listed out in other reviews. ${ }^{7-9,11}$ Some of these methods indeed slow voltage fade down, but fully eliminating it appears impossible (Figure 4e). ${ }^{65,96}$

Lastly, these materials show poor stability when charged to high potentials for gaining extra anionic capacity that eventually triggers oxygen release - an effect briefly touched above. Such a correlation between high activity and poor stability is not astonishing, as the same is witnessed in other electrochemical systems, e.g. water-splitting catalysts. ${ }^{97}$ The typical anionic activation plateau observed during the first charge of Li-rich NMCs, which triggers lattice oxygen release, is an early indicator of instability, and oxygen release could be hypothesized to continue in later cycles, although at a slower rate due to oxygen-blocking surface reconstruction. Hence, there is an impetus for particle-level design strategies, e.g. core-shell or concentration-gradient particles, to protect the material's bulk against oxygen release. Even the disordered rock-salt phases are not stable over long cycling. ${ }^{75,77,80,81}$ So far, the most promising system is the model $\beta$ - $\mathrm{Li}_{2} \mathrm{IrO}_{3}$ phase that allows complete $\mathrm{Li}$ removal, hence highlighting the importance of 3-D structures as a promising future direction for stable anionic redox cathodes. ${ }^{63}$

In summary, the practical viability of Li-rich cathodes is closely tied to the anionic redox process and therefore mitigation strategies must directly target it. The drawbacks of these cathodes do not rule them out completely, as this is just a question of matching the right cathode to the right application.

\section{Perspectives and Conclusions}

Owing to theoretical and experimental advances, the novel anionic redox concept has matured but not enough to yet reach the marketplace. In light of the above-mentioned roadblocks affecting real-world performance of these cathodes, we next evaluate the chances of overcoming these barriers and the positioning of these materials with respect to existing cathodes depending on different figures of merit. 
From a materials perspective, besides continuing the pursuit to chemically improve the promising Li-rich NMC layered oxides, efforts are still required to widen the number of $\mathrm{M}-\mathrm{L}$ couples (with L beyond oxygen) as well as host structures showing reversible anionic redox chemistry. This calls for selecting M-L couples with suitable band positions, a task towards which theory can be of great predictive help if the notion of cationic disorder, which usually accompanies anionic oxidation, could be properly incorporated into the calculations. Inorganic chemistry offers at least two options. As already initiated, one consists in lowering the energy of the TM $d$ states by moving down within the periodic table $(\mathrm{Mn} \rightarrow \mathrm{Ru} \rightarrow \mathrm{Ir})$ and reducing the $U$ term so that they approach the $\mathrm{O} 2 p$ non-bonding states to enhance the chances of triggering anionic redox while lowering $\mathrm{O}_{2}$ release, but at the expense of raw material costs. Another option relies on replacing oxygen with less electronegative ligands, such as $\mathrm{L}=\mathrm{S}, \mathrm{Se}$, or Te, so as to raise the energy of the $\mathrm{L}$ $n p$ non-bonding levels $(n=3$ for $\mathrm{L}=\mathrm{S})$ which now will approach/penetrate the $\mathrm{TM} d$ band, hence the making of various transition metal sulfides (or selenides, tellurides). By returning to the sulfur chemistry, we will of course minimize the chances of releasing $\mathrm{S}_{2}$ owing to its lower chemical hardness as compared to oxygen, but at the expense of a lower voltage. Midway between these two options, oxy-sulfides appear appealing since they could mitigate $\mathrm{O}_{2}$ release while staying high in potential, but so far any attempts to make oxy-sulfides with $3 d$ metals have been unsuccessful with the exception of amorphous $\mathrm{TiO}_{y} \mathrm{~S}_{z}{ }^{67}$ Finally, a more ambitious challenge could be the identification of oxides showing solely anionic redox and a few interesting paths do exist as long as soft chemistry is used to manipulate some of these metastable phases.

Parallel to today's activities on Li-rich NMC, there is an as intense push on NMC materials (Figure 5) with the NMC 622 composition already gaining popularity for electric vehicles. These compositions enable high energy density batteries $\left(>250 \mathrm{Wh}^{\mathrm{kg}_{\text {cell }}}{ }^{-1}\right)$, while showing excellent cycle/calendar life and preserving safety. ${ }^{8,98,99}$ Further increasing the Ni content and minimizing the problematic Co results in even higher energy NMC 811 composition that is foreseen 
to be commercialized by 2021. At that stage, Li-rich NMC will no longer be advantageous concerning low Co content (Figure 5d), unless Co-free Li-rich materials are mastered. Moreover, Li-rich NMCs are intrinsically penalized by a smaller tap-density as compared to NMC phases ${ }^{7}$, hence weakening their advantage when comparing volumetric energy density (Figure 5c). Besides, voltage hysteresis, sluggish kinetics, and voltage fade are other major concerns with Li-rich NMCs since these issues deteriorate respectively the energy efficiency, power density, and cyclability (Figures 5e-g). Concerning voltage fade, chemists are endeavouring via well-selected chemical substitutions to prevent trapping of cations in tetrahedral sites or via surface treatments and the realization of core-shell / concentration-gradient particles. Battery engineers are also optimistic that voltage fade can be overcome via a sophisticated battery management system (BMS). Solving the issues of voltage hysteresis and sluggish anionic kinetics is more challenging. Few approaches are pursued and the most promising one is nested in the design of materials within which cationic and anionic redox processes are not decoupled but occur at the same potential so that cationic redox with fast kinetics can serve as a redox mediator for the sluggish anionic process - a situation offered by the model $\mathrm{Li}_{2} \mathrm{IrO}_{3}$. However, achieving such a specific situation to Li-rich phases made of $3 d$ metals, despite a plethora of work, is still awaited. Overall, these issues could delay the market implementation of Li-rich NMCs in comparison to NMCs because the latter show superiority in numerous figures of merit, except material specific energy (Figure 5).

In summary, through this six years' research journey on anionic-redox-based insertion compounds, we have learned the added value of model systems in not just revealing fundamental insights but also inspiring mitigation strategies that can further be implemented in other technologies beyond $\mathrm{Li}$-ion, such as Na-ion for which anionic redox activity is gaining momentum as well. ${ }^{69,70}$ We have also learned how simple concepts of theoretical chemistry can rationalize a new mechanism and guide in the design of new materials. Our future direction would therefore be to master holistically the underlying thermodynamics and kinetics of anionic redox by bridging the 
learnings between model and practical materials, aided by theory. Such an approach, followed by more complex considerations such as mesoscale inhomogeneities during electrochemical reactions, is essential for taking high-capacity anionic redox cathodes beyond the labs and into the market and the chance of succeeding is high provided we solve the identified limitations in due time. This time constraint is dictated by the rapidly shrinking window of opportunity that was expected early-on for Li-rich NMCs because of steady progresses realized with NMCs. However, hope must prevail since the issues are well-identified and finding solutions will be catalysed by the ever improving synergy between theorists and experimentalists.

\section{Acknowledgements}

The authors are greatly thankful to M.-L. Doublet and M. Saubanère for their openness and patience for lively theoretical discussions, and also to A. Grimaud, A. Abakumov, J. Vergnet, G. Rousse, A Pérez, Q. Jacquet, M. Sathiya, and A. Georges for sharing their knowledge and challenging comments. J.-M.T. and G.A. acknowledge the funding from the European Research Council (ERC) (FP/2014)/ERC Grant-Project 670116-ARPEMA.

\section{Competing Financial Interests}

The authors declare no competing financial interests. 


\section{Figure Captions}

Figure 1 | Crystal structures and electrochemical properties of layered oxides. The structures of layered oxides, such as $\mathrm{LiCoO}_{2}(\mathbf{a})$, and Li-rich layered oxides, such as $\mathrm{Li}_{1.2} \mathrm{Ni}_{0.13} \mathrm{Mn}_{0.54} \mathrm{Co}_{0.13} \mathrm{O}_{2}$ (Li-rich NMC) that is derived from $\mathrm{Li}_{2} \mathrm{MnO}_{3}(\mathbf{b})$, are shown with the latter containing extra $\mathrm{Li}$ within the metal layers. The corresponding voltage profiles (c and $\mathbf{d}$ ) indicate a nearly doubling of capacity and specific energy for the Li-rich phase due to cumulative cationic and anionic redox processes, which take place on charge and discharge as indicated on the electrochemical curves by the nature of ions involved in the redox processes.

Figure 2 | Band structure of oxides and the anionic redox mechanism. As depicted from (a) to (b) to (c), the schematic band structure of transition-metal oxides (c) can be built by extrapolating the molecular orbital energy diagram for octahedral $\mathrm{MO}_{6}(\mathbf{a}) . \mathrm{O}_{\mathrm{NB}}$ denotes the $\mathrm{O} 2 p$ non-bonding states located below the antibonding $(\mathrm{M}-\mathrm{O})^{*}$ band and just above the bonding $(\mathrm{M}-\mathrm{O})$ band. A comparison of $\mathrm{LiMO}_{2}$ (d) and Li-rich $\mathrm{Li}_{2} \mathrm{MO}_{3}$ (e), in terms of their crystal structures (focusing on slabs of $\mathrm{MO}_{2}$ or $\mathrm{Li}_{1 / 3} \mathrm{M}_{2 / 3} \mathrm{O}_{2}$ ) and the relevant parts of their band structures, reveals how the two structures differ in oxygen coordination. Thick black lines highlight three $\mathrm{M}$ neighbours for each $\mathrm{O}$ in $\mathrm{LiMO}_{2}(\mathbf{d})$, compared to only two in the honeycomb-arranged $\mathrm{Li}_{2} \mathrm{MO}_{3}(\mathbf{e})$, thus giving rise to $\mathrm{O}$ $2 p$ non-bonding states in the latter. Note that these are schematic band structures without taking into account electron-electron correlations. Taking Mott-Hubbard splitting into account, the $\mathrm{Li}_{2} \mathrm{MO}_{3}$ band structure is further classified under three cases $(\mathbf{f}-\mathbf{h})$, depending on the interplay between the $d$ - $d$ Coulomb repulsion term $U$ and the charge transfer term $\Delta$. UHB and LHB denote the upper and lower Hubbard bands respectively. $U$ typically ranges from 0 to $6 \mathrm{eV}$. In Case 2 (g), the overlap of LHB and non-bonding $\mathrm{O} 2 p$ states indicates the adequate band positioning for triggering a reversible anionic redox. Electron removal from this scenario leads to a two-band redox process giving extra capacity and is usually followed by $\mathrm{MO}_{6}$ octahedral distortion leading to short $\mathrm{O}-\mathrm{O}$ distances. In contrast, this is not possible in Case 3 (h) that shows irreversible anionic redox, leading to $\mathrm{O}_{2}$ gas release upon electron removal.

Figure 3 | Materials exploration pathway for Li-rich oxides. Cathode materials having anionic redox activity are shown as a function of their $\mathrm{O} / \mathrm{M}$ ratio (top horizontal axis, increasing from left to right) where the local structure around oxygen is displayed in the top row to highlight the increasing amount of $\mathrm{O} 2 p$ non-bonding states when going from $\mathrm{LiMO}_{2}$ to $\mathrm{Li}$-rich compositions of $\mathrm{Li}_{2} \mathrm{MO}_{3}$, $\mathrm{Li}_{3} \mathrm{MO}_{4}$, and so on. The vertical axis on the left simultaneously monitors the dimensionality of the $\mathrm{M}-\mathrm{O}$ network in the long-range structure (decreasing from top to bottom). The best candidates known today can be found within the quadrant delimited by $2.5 \leq \mathrm{O} / \mathrm{M} \leq 4$ and a dimensionality ranging from 2-D to 3-D. Curved dashed lines demarcate the composition-dimensionality boundaries beyond which finding Li-rich materials seems unfeasible. Lastly, the bottom right corner of this plot is rich in known compositions, with most of them unfortunately being unstable against $\mathrm{O}_{2}$ release, besides the fact that their structure consists of isolated $\mathrm{MO}_{y}$ units. 
Figure 4 | Practical challenges facing Li-rich cathodes. The key drawbacks of these materials are shown - namely voltage hysteresis (a) which leads to a lowered energy efficiency, sluggish kinetics (b and c) which prevents high power applications, and voltage fade (d and e) which necessitates complicated BMS systems for tackling it. Note that voltage hysteresis is minimum for model compounds based on heavy $4 d / 5 d$ metals (upper two panels in a) and increases noticeably as we move to Li-rich NMC, then becoming huge for the cation-disordered rock-salt phases, such as $\mathrm{Li}_{1.2} \mathrm{Mn}_{0.4} \mathrm{Ti}_{0.4} \mathrm{O}_{2}{ }^{75}$ (lowest panel in a). Using the model $\mathrm{Li}_{2} \mathrm{Ru}_{0.75} \mathrm{Sn}_{0.25} \mathrm{O}_{3}$ cathode, the sluggish kinetics of anionic redox is revealed (as shown in b) by recording the voltage profiles at increasing C-rates. A large polarization is seen for the anionic reduction peak ${ }^{94}$ that is located at high potential $^{62}$. The cationic/anionic charge compensation is more complicated in Li-rich NMC (c), but the same trend of slow kinetics is observed at potentials where anionic redox activity is found. ${ }^{49}$ Concerning voltage fade, the stabilized voltage profile in the model $\mathrm{Li}_{2} \mathrm{Ru}_{0.75} \mathrm{Sn}_{0.25} \mathrm{O}_{3}$ system is compared against $\mathrm{Li}_{2} \mathrm{Ru}_{0.75} \mathrm{Ti}_{0.25} \mathrm{O}_{3}$ (d) that shows an aggravated voltage fade (marked by thick arrows). ${ }^{73}$ In contrast, a significant voltage fade persists even in a state-of-the-art surface-modified Li-rich NMC (e). ${ }^{65}$ Data for $\mathrm{Li}_{1.2} \mathrm{Mn}_{0.4} \mathrm{Ti}_{0.4} \mathrm{O}_{2}$ in a taken from ref. ${ }^{75}$ (NPG). Panels adapted from: $\mathbf{b}$, ref. $^{94}$ (ECS); d, ref. ${ }^{73}$ (NPG). Panels reproduced from: c, ref. ${ }^{49}$ (NPG); e, ref. ${ }^{65}$ (NPG).

Figure 5 | Benchmarking Li-rich NMC against NMCs. The central spider-chart (a) compares electrode materials in terms of six key figures of merit $(\mathbf{b}-\mathbf{g})$ that are important for practical applications. Material-level specific energy (b) and cell-level energy density (considering graphite anode, $\mathbf{c}$ ) are compared in detail for Li-rich NMC relevant herein with the evolving NMC cathodes $(111 \rightarrow 622 \rightarrow 811)$. Worth noting is that NMC 811 fares equally to Li-rich NMC in terms of cell energy density, a critical metric for electric vehicle applications. While both cathodes are similar in terms of Co content (d), note the poorer energy efficiency of Li-rich NMC (e). Also the Li-rich NMC presently falls short in terms of cyclability (f) and power density (g), the former being based on capacity retention (over 100 cycles in Li half-cells) as well as voltage fade. The Ragone plot in (g) is drawn with a logarithmic scale for specific power. Panels $(\mathbf{b}-\mathbf{e})$ are based on our data and are in accordance with literature. ${ }^{7,98,99}$ The data in (f) for Li-rich NMC (filled symbols) are based on: ref. $^{65}$ (circle); ref. ${ }^{96}$ (diamond); ref. ${ }^{100}$ (triangle); and for NMC 622 (unfilled circles) and NMC 811 (unfilled triangles) on ref. ${ }^{99}$. The data in (g) for Li-rich NMC (filled symbols) are based on: ref. ${ }^{100}$ (circles); ref. ${ }^{101}$ (diamonds), ref. ${ }^{102}$ (triangles); and for NMC 811 (unfilled symbols) on: ref. $^{103}$ (circles), ref. ${ }^{104}$ (diamonds), ref. ${ }^{105}$ (triangles). For the sake of comparison, other anionic redox cathodes, such as layered $\mathrm{Li}_{2} \mathrm{Ru}_{0.75} \mathrm{Sn}_{0.25} \mathrm{O}_{3}$ (LRSO) and disordered rock-salt $\mathrm{Li}_{1.2} \mathrm{Mn}_{0.4} \mathrm{Ti}_{0.4} \mathrm{O}_{2}(\mathrm{Li}$ $\mathrm{Mn}-\mathrm{Ti}-\mathrm{O})^{75}$ are also included in $(\mathbf{b}-\mathbf{e})$. Overall, this spider-chart indicates that the enthusiastic expectations generated by the Li-rich NMC phases due to their outstanding specify energy must be reconsidered, as further work is needed on other frontiers. 


\section{Box 1 Key steps in the emergence of anionic redox chemistry.}

- 1990s. It was explained using schematic band diagrams shown in panel a that in highly covalent chalcogenides, the transition-metal $d$ band penetrates into the ligand $s p$ band so that part of the $s p$ electrons are poured into the $d$ band - leaving behind holes - hence the terminology ligand-hole chemistry. ${ }^{20,21}$

- 1999. The onset of oxygen redox was proposed at high potential in $\mathrm{Li}_{x} \mathrm{CoO}_{2}$ based on the observation of slightly shortened $\mathrm{O}-\mathrm{O}$ distances as deduced by synchrotron diffraction ${ }^{25}$ together with magnetic studies ${ }^{24}$. Such an oxygen activity was simultaneously endorsed by theorists via first-principle calculation of electrondensity maps shown in panel $b^{26,27}$

- 2002-2008. Direct spectroscopic measurements of the ligand confirmed oxygen redox-activity in $\mathrm{Li}_{x} \mathrm{CoO}_{2}$ (through $\mathrm{O}$ K-edge $\mathrm{XAS}^{28}$ and $\mathrm{O} 1 s \mathrm{XPS}^{29}$ ) and sulfur redox in $\mathrm{Li}_{x} \mathrm{TiO}_{y} \mathrm{~S}_{z}$ (through $\mathrm{S} 2 p$ core-level XPS ${ }^{67}$ ).

- 2002-2007. Early reports of electrochemical activity in $\mathrm{Li}_{2} \mathrm{MnO}_{3}{ }^{34}$ were followed by the works of several prominent research groups leading to the discovery of high capacity Li-rich $\mathrm{NMCs}^{36,37}$, which can be visualized on a ternary phase diagram (panel c) as combinations of $\mathrm{Li}_{2} \mathrm{MnO}_{3}$ and $\mathrm{LiMO}_{2}$ end-members.

\section{insert Figure here}

- 2013-2015. The reversible activity of lattice oxygen was proposed in Li-rich NMC based on a series of characterizations ${ }^{6,44,45}$, notably operando XAS measurement of transition-metal K-edges which indirectly showed that solely cationic redox was insufficient to account for the overall charge compensation.

- 2013-2015. Model Li-rich phases $\left(\mathrm{Li}_{2} \mathrm{MO}_{3}\right.$ with $\mathrm{M}$ being $\mathrm{Ru}$ and $\mathrm{Ir}$ ), which are isostructural with Li-rich NMC and likewise show the typical staircase-like charge and sloped discharge (panel d), were designed. ${ }^{5,55}$ Using XPS and EPR, the appearance of $\left(\mathrm{O}_{2}\right)^{n-}$ species on charging was first proven, prior to directly visualizing $\mathrm{O}-\mathrm{O}$ dimers using TEM and neutron diffraction.

- 2016. Reversible oxygen activity was experimentally proven in Lirich NMC through an arsenal of characterization techniques, with notably the use of ${ }^{18} \mathrm{O}$-labelled operando mass spectrometry to quantify the amount of lattice oxygen released as $\mathrm{O}_{2}$ and $\mathrm{CO}_{2}$ during first charge. $^{46}$

- 2016. The anionic redox mechanism was theoretically rationalized in different types of $3 d, 4 d$, and $5 d$ metal-based cathodes, laying out the importance of $\mathrm{O} 2 p$ non-bonding states (or $\mathrm{Li}-\mathrm{O}-\mathrm{Li}$ configurations in panel e, top), the conditions for $\mathrm{O}-\mathrm{O}$ shortening, and the reductive coupling mechanism (panel e, bottom) to achieve reversible anionic redox. ${ }^{51-53}$

Figure. Panels adapted from: d, ref. ${ }^{5}$ (NPG); e, refs. ${ }^{5,51}$ (NPG). Panels reproduced from: a, ref. ${ }^{20}$ (Wiley); b, ref. ${ }^{26}$ (APS); c, ref. $^{37}(\mathrm{RSC})$. 


\section{Box 2 | New paradigms in characterization techniques for anionic redox.}

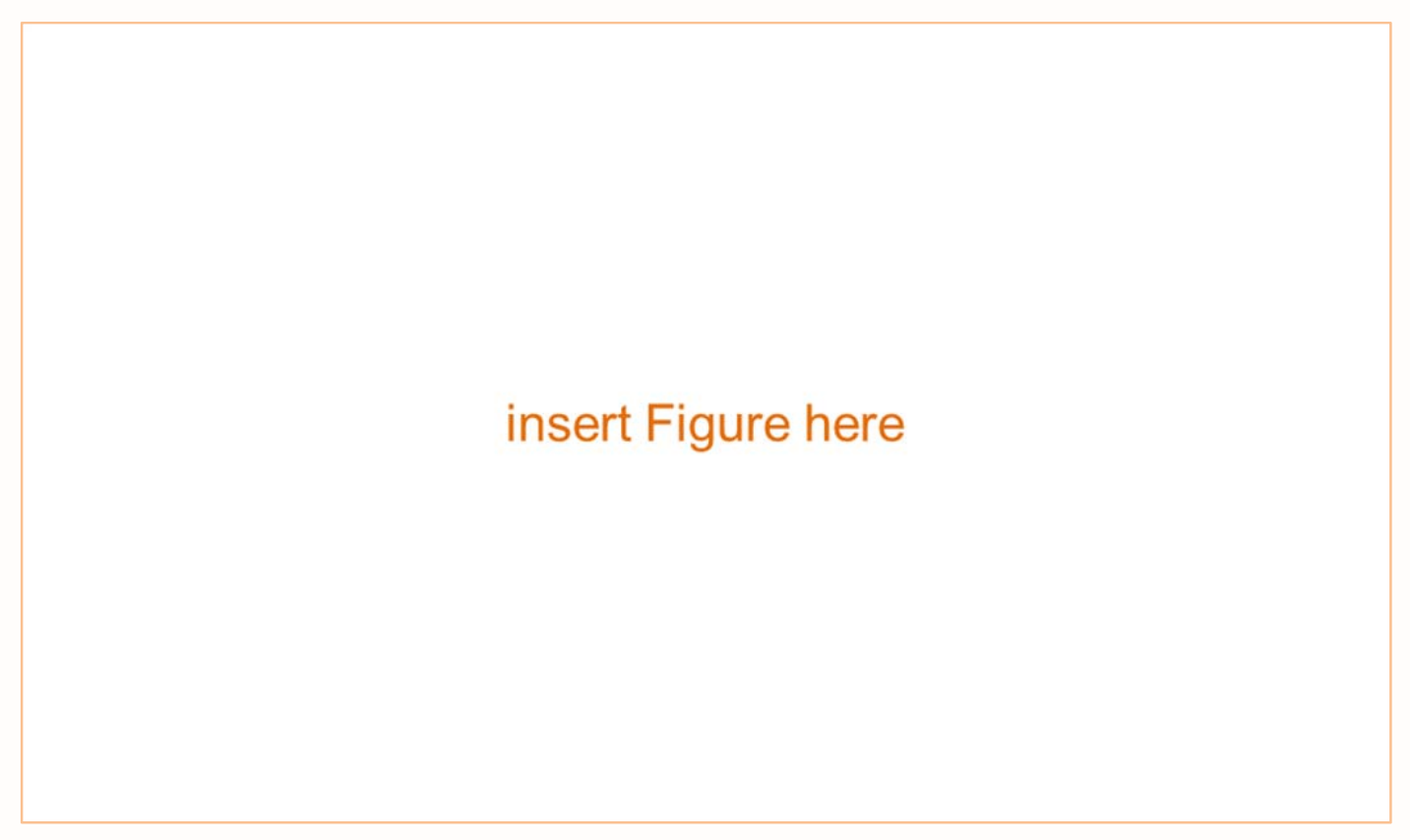

Figure. Panels reproduced from: a and c, ref. ${ }^{55}$ (AAAS); b, ref. ${ }^{63}$ (NPG); d, ref. ${ }^{49}$ (NPG); e, ref. ${ }^{54}$ (NPG); f, ref. ${ }^{50}$ (NPG); g, ref. $^{62}$ (ACS).

Characterizing anionic redox calls for specific structural and spectroscopic techniques that often require access to large instruments. ${ }^{106}$

Structural characterization. Neutron diffraction is very sensitive to light elements and can accurately assess the atomic positions within a structure, hence allowing the quantification of interatomic distances to spot the $\mathrm{O}-\mathrm{O}$ dimers, provided that such species exhibit long-range ordering and that stacking faults are at a minimum. Panel a shows the refined crystal-structure of $\alpha-\mathrm{Li}_{2-x} \mathrm{IrO}_{3}$ for $x=1.5$ and panel $\mathrm{b}$ shows the distorted $\mathrm{IrO}_{6}$ octahedra in $\beta-\mathrm{Li}_{2-x} \mathrm{IrO}_{3}$ for $x=1.0$. ${ }^{55,63}$ Electron diffraction and annular bright field scanning transmission electron microscopy (ABF-STEM) are elegant ways to directly visualize the $\mathrm{O}-\mathrm{O}$ shortening, as shown for $\alpha-\mathrm{Li}_{2-x} \mathrm{IrO}_{3}, x=1.5$ in panel c. ${ }^{55} \mathrm{ABF}-\mathrm{STEM}$ imaging requires high sample-stability against electron-beam irradiation, unlike electron diffraction that can be applied to more fragile structures that are typically obtained after charging. An ultimate goal would be to combine STEM with electron energy loss spectroscopy (EELS), in order to map the electronic states of individual oxygen and TM atoms. Note that the above techniques are suitable only for crystalline materials, whereas $\mathrm{O}-\mathrm{O}$ distance determination is still awaited in less-ordered materials via local structural techniques, such as pair distribution function (PDF) analysis of diffraction data.

Characterizing the charge compensation mechanism. A powerful technique for measuring changes in the ligand's electronic state for a variety of battery materials is X-ray photoelectron spectroscopy (XPS). ${ }^{29,48,67}$ However, it remains surface-sensitive. This drawback can partially be overcome with hard-XPS (HAXPES), through which higher energy synchrotron X-rays can reveal bulk information (panel d shows $\mathrm{O}^{n-}$ species in fully-charged Li-rich NMC). ${ }^{49}$ Note that HAXPES can only detect the $\mathrm{O}^{n-}$ species, without the possibility to further reveal its nature. Moreover, operando HAXPES is yet to be developed. On the other hand, electron paramagnetic resonance (EPR) spectroscopy, which detects radical or unpaired-spin species, is bulk-sensitive with operando capabilities for quantitatively probing the $\left(\mathrm{O}_{2}\right)^{n-}$ species as well as for visualizing their nucleation via EPR imaging whose spatial resolution needs further improvement (panel e shows the $1^{\text {st }}$ discharge process of $\left.\mathrm{Li}_{2} \mathrm{Ru}_{0.75} \mathrm{Sn}_{0.25} \mathrm{O}_{3}\right)^{54}$. EPR requires that the sample is not exceedingly metallic, the interfering signals from transition-metals are absent, and the $\left(\mathrm{O}_{2}\right)^{n-}$ species are EPR-active. Therefore, complementary characterizations may be needed.

Soft X-ray absorption spectroscopy (soft-XAS) at the O K-edge is also being used frequently. ${ }^{46,47,75}$ However, a common pitfall is encountered when correlating the intensity changes in O K-edge XAS spectra with the holes on oxygen, because of $\mathrm{O}-2 p / \mathrm{TM}-\mathrm{n} d$ hybridization. ${ }^{106}$ Moreover, the choice of detection mode is crucial for obtaining true bulk information, for which soft-XAS-based scanning transmission X-ray microscopy (STXM) has emerged as a more suitable method for revealing the redox mechanisms along with particle-level inhomogeneities (panel f shows the bulk sensitivity of 
STXM and its ability to spot the signature of anionic redox in Li-rich NMC, as marked by red arrow). ${ }^{50}$ Further theoretical developments are awaited to fully interpret the O K-edge XAS spectra. In contrast, soft-XAS measurements of TM L-edges provide unambiguously their oxidation states ${ }^{107}$, which can then indirectly be used to discuss the anionic redox activity ${ }^{75,77}$. Resonant inelastic X-ray scattering (RIXS) can also characterize oxygen holes, as neatly done in rare-earth nickelates ${ }^{32}$. A handful of RIXS measurements on Li-rich cathodes are already reported ${ }^{46,50}$, but more rigorous implementations are awaited. On the other hand, operando measurement of TM K-edges with hard-XAS is technically simpler. ${ }^{45,56}$ Moreover, when performed in transmission mode encompassing an ensemble of particles, it can provide an accurate quantification of change compensation (panel g shows the deconvoluted $5^{\text {th }}$ cycle of $\mathrm{Li}_{2} \mathrm{Ru}_{0.75} \mathrm{Sn}_{0.25} \mathrm{O}_{3}$ ). ${ }^{62}$

Lastly, one can experimentally reconstruct the density of states of Li-rich cathodes near $E_{\mathrm{F}}$, as recently achieved for perovskite-oxides and $3 d$-metal fluorides, by combining XAS, X-ray emission spectroscopy (XES), and valence-band XPS. ${ }^{106}$ The $U$ and $\Delta$ terms can thus be quantified, leading to a harmonization of the theoretical framework governing the anionic redox chemistry that was presented in Figure 2. 


\section{Box 3 | How to evaluate the practicability of novel anionic redox cathodes?}

Anionic redox cathodes show unique electrochemical properties, distinguishing them from classical insertion cathodes, which must be evaluated consistently for the sake of correct comparison and practical evaluation.

1. Voltage hysteresis. Voltage profiles should be measured at a low C-rate (slower than $\mathrm{C} / 50$ ) or with GITT/PITT methods to check for a quasi-thermodynamic path-dependence. In this case, the $d Q / d V$ profiles will not mirror between charge vs. discharge, and voltage window experiments ${ }^{49,87}$ are recommended. Moreover, the lowered energy efficiency ${ }^{90}$ should be reported.

2. Electrochemical Kinetics. The charge-transfer resistance and Li diffusion coefficient should be measured as a function of Li content via three-electrode impedance or other electroanalytical methods, and checked whether sluggish kinetics / transport accompanies anionic redox. ${ }^{49,94}$ Besides, recording $d Q / d V$ profiles at different C-rates (e.g. Figure $\left.4 \mathrm{~b}\right)$ may provide a simple visual cue for sluggish kinetics of certain redox peaks.

3. Cyclability. Capacity retention, a common metric for cyclability, can be remarkable for anionic redox cathodes such as optimized Li-rich NMCs, but it does not automatically imply voltage stabilization. ${ }^{65,96,100}$ Therefore, voltage fade should be reported as average charge and discharge voltages vs. cycle number, in accordance with previous recommendations. ${ }^{10,95}$ Furthermore, while comparing different mitigation strategies, lets recall that some treatments unintentionally lead to capacity reduction (thereby lower extent of delithiation) and hence voltage fade 'appears' to ameliorate, but in reality it is just because the material is not being oxidized fully. 


\section{References}

1. Tarascon, J.-M. \& Armand, M. Issues and challenges facing rechargeable lithium batteries. Nature 414, 359-367 (2001).

2. Schmidt, O., Hawkes, A., Gambhir, A. \& Staffell, I. The future cost of electrical energy storage based on experience rates. Nat. Energy 6, 17110 (2017).

3. 'This is what we die for': human rights abuses in the Democratic Republic of the Congo power the global trade in cobalt. (Amnesty International, Index: AFR 62/3183/2016, 2016).

4. Sathiya, M. et al. High Performance $\mathrm{Li}_{2} \mathrm{Ru}_{1-y} \mathrm{Mn}_{y} \mathrm{O}_{3}(0.2 \leq y \leq 0.8)$ Cathode Materials for Rechargeable Lithium-Ion Batteries: Their Understanding. Chem. Mater. 25, 1121-1131 (2013).

5. Sathiya, M. et al. Reversible anionic redox chemistry in high-capacity layered-oxide electrodes. Nat. Mater. 12, 827-835 (2013).

Using a model compound derived from $\mathrm{Li}_{2} \mathrm{RuO}_{3}$, this work demonstrated reversible anionic redox as a viable approach for energy storage.

6. Koga, H. et al. Reversible oxygen participation to the redox processes revealed for Li1. 20Mn0. 54Co0. 13Ni0. 13O2. J. Electrochem. Soc. 160, A786-A792 (2013).

7. Zheng, J. et al. Li- and Mn-Rich Cathode Materials: Challenges to Commercialization. Adv. Energy Mater. 1601284 (2016). doi:10.1002/aenm.201601284

8. Li, W., Song, B. \& Manthiram, A. High-voltage positive electrode materials for lithium-ion batteries. Chem Soc $\operatorname{Rev}$ (2017). doi:10.1039/C6CS00875E

9. Hy, S. et al. Performance and design considerations for lithium excess layered oxide positive electrode materials for lithium ion batteries. Energy Environ. Sci. 9, 1931-1954 (2016).

10. Croy, J. R., Balasubramanian, M., Gallagher, K. G. \& Burrell, A. K. Review of the U.S. Department of Energy’s 'Deep Dive' Effort to Understand Voltage Fade in Li- and Mn-Rich Cathodes. Acc. Chem. Res. 48, 2813-2821 (2015).

This review paper summarizes the research carried out at Argonne Labs. (USA) on understanding the fundamental mechanisms behind voltage fade and voltage hysteresis in Li-rich NMCs.

11. Hong, J. et al. Review-Lithium-Excess Layered Cathodes for Lithium Rechargeable Batteries. J. Electrochem. Soc. 162, A2447-A2467 (2015).

12. Rozier, P. \& Tarascon, J. M. Review-Li-Rich Layered Oxide Cathodes for Next-Generation Li-Ion Batteries: Chances and Challenges. J. Electrochem. Soc. 162, A2490-A2499 (2015).

13. Grimaud, A., Hong, W. T., Shao-Horn, Y. \& Tarascon, J.-M. Anionic redox processes for electrochemical devices. Nat. Mater. 15, 121-126 (2016).

14. Yabuuchi, N. Solid-state Redox Reaction of Oxide Ions for Rechargeable Batteries. Chem. Lett. 46, 412-422 (2017).

15. Li, B. \& Xia, D. Anionic Redox in Rechargeable Lithium Batteries. Adv. Mater. 1701054 (2017). doi:10.1002/adma.201701054

16. Goodenough, J. B. \& Kim, Y. Challenges for Rechargeable Li Batteries. Chem. Mater. 22, 587-603 (2010).

17. Whittingham, M. S. Electrical Energy Storage and Intercalation Chemistry. Science 192, 1126-1127 (1976).

18. Lazzari, M. \& Scrosati, B. A Cyclable Lithium Organic Electrolyte Cell Based on Two Intercalation Electrodes. J. Electrochem. Soc. 127, 773-774 (1980).

19. Ozawa, K. Lithium-ion rechargeable batteries with $\mathrm{LiCoO} 2$ and carbon electrodes: the LiCoO2/C system. Solid State Ion. 69, 212-221 (1994).

20. Rouxel, J. Anion-Cation Redox Competition and the Formation of New Compounds in Highly Covalent Systems. Chem. - Eur. J. 2, 1053-1059 (1996).

21. Rouxel, J. Some solid state chemistry with holes: Anion-cation redox competition in solids. Curr. Sci. 73, 31-39 (1997).

This seminal review of anionic redox mechanisms in transition-metal chalcogenides demonstrates the versatile impact of such chemistry on structure, synthesis, and properties.

22. Bichat, M.-P. et al. Redox-Induced Structural Change in Anode Materials Based on Tetrahedral (MPn4)xTransition Metal Pnictides. Chem. Mater. 16, 1002-1013 (2004).

23. Amatucci, G. G., Tarascon, J. M. \& Klein, L. C. CoO2, The End Member of the Li x CoO2 Solid Solution. J. 
Electrochem. Soc. 143, 1114-1123 (1996).

24. Amatucci, G. G. PhD dissertation. (Rutgers University, 1995).

25. Tarascon, J. M. et al. In Situ Structural and Electrochemical Study of Ni1-xCoxO2 Metastable Oxides Prepared by Soft Chemistry. J. Solid State Chem. 147, 410-420 (1999).

26. Aydinol, M. K. et al. Ab initio study of lithium intercalation in metal oxides and metal dichalcogenides. Phys. Rev. B 56, 1354 (1997).

27. Ceder, G. et al. Identification of cathode materials for lithium batteries guided by first-principles calculations. Nature 392, 694 (1998).

28. Yoon, W.-S. et al. Oxygen Contribution on Li-Ion Intercalation-Deintercalation in $\mathrm{LiCoO}_{2}$ Investigated by O KEdge and Co L-Edge X-ray Absorption Spectroscopy. J. Phys. Chem. B 106, 2526-2532 (2002).

29. Dahéron, L. et al. Electron Transfer Mechanisms upon Lithium Deintercalation from $\mathrm{LiCoO}_{2}$ to $\mathrm{CoO}_{2}$ Investigated by XPS. Chem. Mater. 20, 583-590 (2008).

30. Sarma, D. D., Sreedhar, K., Ganguly, P. \& Rao, C. N. R. Photoemission study of YBa2Cu3O7 through the superconducting transition: Evidence for oxygen dimerization. Phys. Rev. B 36, 2371-2373 (1987).

31. Demourgues, A. et al. Additional Oxygen Ordering in 'La2NiO4.25' (La8Ni4O17): II. Structural Features. $J$. Solid State Chem. 106, 330-338 (1993).

32. Bisogni, V. et al. Ground-state oxygen holes and the metal-insulator transition in the negative charge-transfer rare-earth nickelates. Nat. Commun. 7, ncomms13017 (2016).

33. Kalyani, P., Chitra, S., Mohan, T. \& Gopukumar, S. Lithium metal rechargeable cells using Li2MnO3 as the positive electrode. J. Power Sources 80, 103-106 (1999).

34. Robertson, A. D. \& Bruce, P. G. Mechanism of Electrochemical Activity in Li2MnO3. Chem. Mater. 15, 19841992 (2003).

35. Yu, D. Y. W., Yanagida, K., Kato, Y. \& Nakamura, H. Electrochemical Activities in Li2MnO3. J. Electrochem. Soc. 156, A417-A424 (2009).

36. Lu, Z. \& Dahn, J. R. Understanding the Anomalous Capacity of Li/Li[Ni[sub x] Li[sub (1/3-2x/3)]Mn[sub $(2 / 3-x / 3)]] \mathrm{O}[\mathrm{sub} 2]$ Cells Using In Situ X-Ray Diffraction and Electrochemical Studies. J. Electrochem. Soc. 149, A815 (2002).

37. M. Thackeray, M. et al. Li $2 \mathrm{MnO} 3$-stabilized LiMO $2(\mathrm{M}=\mathrm{Mn}, \mathrm{Ni}, \mathrm{Co})$ electrodes for lithium-ion batteries. $J$. Mater. Chem. 17, 3112-3125 (2007).

38. Armstrong, A. R. et al. Demonstrating Oxygen Loss and Associated Structural Reorganization in the Lithium Battery Cathode Li[Ni0.2Li0.2Mn0.6]O2. J. Am. Chem. Soc. 128, 8694-8698 (2006).

39. Boulineau, A. et al. First Evidence of Manganese-Nickel Segregation and Densification upon Cycling in Li-Rich Layered Oxides for Lithium Batteries. Nano Lett. 13, 3857-3863 (2013).

40. Tran, N. et al. Mechanisms Associated with the 'Plateau' Observed at High Voltage for the Overlithiated Li1.12(Ni0.425Mn0.425Co0.15)0.88O2 System. Chem. Mater. 20, 4815-4825 (2008).

41. Hy, S. et al. Direct In situ Observation of Li2O Evolution on Li-Rich High-Capacity Cathode Material, $\operatorname{Li}[\operatorname{NixLi}(1-2 \mathrm{x}) / 3 \mathrm{Mn}(2-\mathrm{x}) / 3] \mathrm{O} 2(0 \leq \mathrm{x} \leq 0.5)$. J. Am. Chem. Soc. 136, 999-1007 (2014).

42. Muhammad, S. et al. Evidence of reversible oxygen participation in anomalously high capacity Li- and Mn-rich cathodes for Li-ion batteries. Nano Energy 21, 172-184 (2016).

43. Yabuuchi, N. et al. Detailed Studies of a High-Capacity Electrode Material for Rechargeable Batteries, Li ${ }_{2} \mathrm{MnO}$ ${ }_{3}-\mathrm{LiCo}_{1 / 3} \mathrm{Ni}_{1 / 3} \mathrm{Mn}_{1 / 3} \mathrm{O}_{2}$. J. Am. Chem. Soc. 133, 4404-4419 (2011).

44. Koga, H. et al. Different oxygen redox participation for bulk and surface: A possible global explanation for the cycling mechanism of Li1.20Mn0.54Co0.13Ni0.13O2. J. Power Sources 236, 250-258 (2013).

45. Koga, H. et al. Operando X-ray Absorption Study of the Redox Processes Involved upon Cycling of the Li-Rich Layered Oxide $\mathrm{Li}_{1.20} \mathrm{Mn}_{0.54} \mathrm{Co}_{0.13} \mathrm{Ni}_{0.13} \mathrm{O}_{2}$ in Li Ion Batteries. J. Phys. Chem. C 118, 5700-5709 (2014).

46. Luo, K. et al. Charge-compensation in 3d-transition-metal-oxide intercalation cathodes through the generation of localized electron holes on oxygen. Nat. Chem. 8, 684-691 (2016).

This work demonstrated the reversibility of anionic redox in Li-rich NMC, notably by experimentally showing that the amount of oxygen release was much less than previously believed.

47. Oishi, M. et al. Direct observation of reversible charge compensation by oxygen ion in Li-rich manganese layered oxide positive electrode material, Li1.16Ni0.15Co0.19Mn0.50O2. J. Power Sources 276, 89-94 (2015).

48. Foix, D. et al. X-ray Photoemission Spectroscopy Study of Cationic and Anionic Redox Processes in HighCapacity Li-Ion Battery Layered-Oxide Electrodes. J. Phys. Chem. C 120, 862-874 (2016). 
49. Assat, G. et al. Fundamental interplay between anionic/cationic redox governing the kinetics and thermodynamics of lithium-rich cathodes. Nat. Commun. 8, (2017).

This work proved redox reactivity of bulk lattice oxygen in Li-rich NMC using hard-XPS and further correlated it with the issues of hysteresis and sluggish kinetics.

50. Gent, W. E. et al. Coupling between oxygen redox and cation migration explains unusual electrochemistry in lithium-rich layered oxides. Nat. Commun. 8, (2017).

This work proved redox reactivity of bulk lattice oxygen in Li-rich NMC using STXM and further correlated it with cation migration to explain voltage hysteresis.

51. Seo, D.-H. et al. The structural and chemical origin of the oxygen redox activity in layered and cation-disordered Li-excess cathode materials. Nat. Chem. (2016). doi:10.1038/nchem.2524

This work provided the theoretical rationale for understanding the anionic redox activity in different types of structures.

52. Saubanère, M., McCalla, E., Tarascon, J.-M. \& Doublet, M.-L. The intriguing question of anionic redox in highenergy density cathodes for Li-ion batteries. Energy Env. Sci 9, 984-991 (2016).

This work provided the theoretical rationale for understanding the reversible vs. irreversible anionic redox activity in layered oxides.

53. Xie, Y., Saubanère, M. \& Doublet, M.-L. Requirements for reversible extra-capacity in Li-rich layered oxides for Li-ion batteries. Energy Env. Sci 10, 266-274 (2017).

54. Sathiya, M. et al. Electron paramagnetic resonance imaging for real-time monitoring of Li-ion batteries. Nat. Commun. 6, 6276 (2015).

55. McCalla, E. et al. Visualization of O-O peroxo-like dimers in high-capacity layered oxides for Li-ion batteries. Science 350, 1516-1521 (2015).

This work directly imaged the structural consequence of anionic redox activity, i.e. O-O dimerization, in a model compound based on $\mathrm{Li}_{2} \mathrm{IrO}_{3}$.

56. Li, B. et al. Understanding the Stability for Li-Rich Layered Oxide $\mathrm{Li}_{2} \mathrm{RuO}_{3}$ Cathode. Adv. Funct. Mater. 26, 1330-1337 (2016).

57. Okubo, M. \& Yamada, A. Molecular Orbital Principles of Oxygen-Redox Battery Electrodes. ACS Appl. Mater. Interfaces (2017). doi:10.1021/acsami.7b09835

58. Zaanen, J., Sawatzky, G. A. \& Allen, J. W. Band gaps and electronic structure of transition-metal compounds. Phys. Rev. Lett. 55, 418-421 (1985).

59. Strehle, B. et al. The Role of Oxygen Release from Li-and Mn-Rich Layered Oxides during the First Cycles Investigated by On-Line Electrochemical Mass Spectrometry. J. Electrochem. Soc. 164, A400-A406 (2017).

60. McCalla, E. et al. Reversible Li-Intercalation through Oxygen Reactivity in Li-Rich Li-Fe-Te Oxide Materials. $J$. Electrochem. Soc. 162, A1341-A1351 (2015).

61. McCalla, E. et al. Understanding the Roles of Anionic Redox and Oxygen Release during Electrochemical Cycling of Lithium-Rich Layered $\mathrm{Li}_{4} \mathrm{FeSbO}_{6}$. J. Am. Chem. Soc. 137, 4804-4814 (2015).

62. Assat, G. et al. Decoupling Cationic-Anionic Redox Processes in a Model Li-rich Cathode via Operando X-ray Absorption Spectroscopy. Chem. Mater. (2017). doi:10.1021/acs.chemmater.7b03434

63. Pearce, P. E. et al. Evidence for anionic redox activity in a tridimensional-ordered Li-rich positive electrode $\beta$ Li2IrO3. Nat. Mater. 16, 580-586 (2017).

64. Kim, S. et al. Material design of high-capacity Li-rich layered-oxide electrodes: Li 2 MnO 3 and beyond. Energy Environ. Sci. (2017). doi:10.1039/C7EE01782K

65. Qiu, B. et al. Gas-solid interfacial modification of oxygen activity in layered oxide cathodes for lithium-ion batteries. Nat. Commun. 7, 12108 (2016).

66. Wu, Z. Y. et al. Sulfur K-Edge X-Ray-Absorption Study of the Charge Transfer upon Lithium Intercalation into Titanium Disulfide. Phys. Rev. Lett. 77, 2101-2104 (1996).

67. Lindic, M. H. et al. XPS investigations of TiOySz amorphous thin films used as positive electrode in lithium microbatteries. Solid State Ion. 176, 1529-1537 (2005).

68. Jobic, S. et al. Crystal and electronic band structure of IrTe2: Evidence of anionic bonds in a CdI2-like arrangement. Z. Für Anorg. Allg. Chem. 598, 199-215 (1991).

69. Yabuuchi, N. et al. A new electrode material for rechargeable sodium batteries: P2-type Na 2/3 [Mg $0.28 \mathrm{Mn}$ 0.72 ]O 2 with anomalously high reversible capacity. J. Mater. Chem. A 2, 16851-16855 (2014).

70. Zhao, C. et al. Review on anionic redox for high-capacity lithium- and sodium-ion batteries. J. Phys. Appl. Phys. 
50, 183001 (2017).

71. Qiu, B. et al. Understanding and Controlling Anionic Electrochemical Activity in High-Capacity Oxides for Next Generation Li-Ion Batteries. Chem. Mater. 29, 908-915 (2017).

72. Sathiya, M. et al. Li 4 NiTeO 6 as a positive electrode for Li-ion batteries. Chem. Commun. 49, 11376-11378 (2013).

73. Sathiya, M. et al. Origin of voltage decay in high-capacity layered oxide electrodes. Nat. Mater. 14, 230-238 (2014).

74. Moore, G. J., Johnson, C. S. \& Thackeray, M. M. The electrochemical behavior of xLiNiO2 $(1-x) \mathrm{Li} 2 \mathrm{RuO} 3$ and Li2Ru1-yZryO3 electrodes in lithium cells. J. Power Sources 119, 216-220 (2003).

75. Yabuuchi, N. et al. Origin of stabilization and destabilization in solid-state redox reaction of oxide ions for lithium-ion batteries. Nat. Commun. 7, 13814 (2016).

76. Glazier, S. L. et al. Characterization of Disordered Li(1+x)Ti2xFe(1-3x)O2 as Positive Electrode Materials in LiIon Batteries Using Percolation Theory. Chem. Mater. 27, 7751-7756 (2015).

77. Yabuuchi, N. et al. High-capacity electrode materials for rechargeable lithium batteries: $\mathrm{Li}_{3} \mathrm{NbO}_{4}$-based system with cation-disordered rocksalt structure. Proc. Natl. Acad. Sci. 112, 7650-7655 (2015).

78. Yabuuchi, N. et al. Synthesis and Electrochemical Properties of Li4MoO5-NiO Binary System as Positive Electrode Materials for Rechargeable Lithium Batteries. Chem. Mater. 28, 416-419 (2016).

79. Matsuhara, T. et al. Synthesis and Electrode Performance of $\mathrm{Li}_{4} \mathrm{MoO}_{5}-\mathrm{LiFeO}_{2}$ Binary System as Positive Electrode Materials for Rechargeable Lithium Batteries. Electrochemistry 84, 797-801 (2016).

80. Lee, J. et al. A new class of high capacity cation-disordered oxides for rechargeable lithium batteries: Li-Ni-TiMo oxides. Energy Environ. Sci. 8, 3255-3265 (2015).

81. Freire, M. et al. A new active Li-Mn-O compound for high energy density Li-ion batteries. Nat. Mater. 15, 173177 (2016).

82. Lee, J. et al. Unlocking the Potential of Cation-Disordered Oxides for Rechargeable Lithium Batteries. Science 343, 519-522 (2014).

83. Perez, A. J. et al. Approaching the limits of cationic and anionic electrochemical activity with the Li-rich layered rocksalt Li3IrO4. Nat. Energy 2, 954-962 (2017).

84. Jacquet, Q. et al. The Li3RuyNb1-yO4 $(0 \leq \mathrm{y} \leq 1)$ System: Structural Diversity and Li Insertion and Extraction Capabilities. Chem. Mater. 29, 5331-5343 (2017).

85. Yamada, A. et al. A New Sealed Lithium-Peroxide Battery with a Co-Doped $\mathrm{Li}_{2} \mathrm{O}$ Cathode in a Superconcentrated Lithium Bis(fluorosulfonyl)amide Electrolyte. Sci. Rep. 4, 5684 (2014).

86. Zhu, Z. et al. Anion-redox nanolithia cathodes for Li-ion batteries. Nat. Energy 1, 16111 (2016).

87. Croy, J. R. et al. Examining Hysteresis in Composite $x \mathrm{Li}_{2} \mathrm{MnO}_{3} \cdot(1-x) \mathrm{LiMO}_{2}$ Cathode Structures. J. Phys. Chem. C 117, 6525-6536 (2013).

88. Wu, Y. et al. Probing the initiation of voltage decay in Li-rich layered cathode materials at the atomic scale. $J$. Mater. Chem. A 3, 5385-5391 (2015).

89. Dees, D. W. et al. Electrochemical Modeling and Performance of a Lithium- and Manganese-Rich Layered Transition-Metal Oxide Positive Electrode. J. Electrochem. Soc. 162, A559-A572 (2015).

90. Meister, P. et al. Best Practice: Performance and Cost Evaluation of Lithium Ion Battery Active Materials with Special Emphasis on Energy Efficiency. Chem. Mater. 28, 7203-7217 (2016).

91. Dogan, F. et al. Re-entrant Lithium Local Environments and Defect Driven Electrochemistry of Li- and Mn-Rich Li-Ion Battery Cathodes. J. Am. Chem. Soc. 137, 2328-2335 (2015).

92. Rinaldo, S. G. et al. Physical Theory of Voltage Fade in Lithium-and Manganese-Rich Transition Metal Oxides. J. Electrochem. Soc. 162, A897-A904 (2015).

93. Konishi, H. et al. Origin of hysteresis between charge and discharge processes in lithium-rich layer-structured cathode material for lithium-ion battery. J. Power Sources 298, 144-149 (2015).

94. Assat, G., Delacourt, C., Corte, D. A. D. \& Tarascon, J.-M. Editors' Choice-Practical Assessment of Anionic Redox in Li-Rich Layered Oxide Cathodes: A Mixed Blessing for High Energy Li-Ion Batteries. J. Electrochem. Soc. 163, A2965-A2976 (2016).

95. Bettge, M. et al. Voltage Fade of Layered Oxides: Its Measurement and Impact on Energy Density. $J$. Electrochem. Soc. 160, A2046-A2055 (2013).

96. Kim, S. et al. A stable lithium-rich surface structure for lithium-rich layered cathode materials. Nat. Commun. 7, 13598 (2016). 
97. Stamenkovic, V. R., Strmcnik, D., Lopes, P. P. \& Markovic, N. M. Energy and fuels from electrochemical interfaces. Nat. Mater. 16, 57-69 (2016).

98. Myung, S.-T. et al. Nickel-rich Layered Cathode Materials for Automotive Lithium-ion Batteries: Achievements and Perspectives. ACS Energy Lett. (2016). doi:10.1021/acsenergylett.6b00594

99. Yoon, C. S. et al. High-Energy Ni-Rich Li[NixCoyMn1-x-y]O2 Cathodes via Compositional Partitioning for Next-Generation Electric Vehicles. Chem. Mater. 29, 10436-10445 (2017).

100. Martha, S. K., Nanda, J., Veith, G. M. \& Dudney, N. J. Electrochemical and rate performance study of highvoltage lithium-rich composition: Li1.2Mn0.525Ni0.175Co0.1O2. J. Power Sources 199, 220-226 (2012).

101. Zheng, J. et al. Electrochemical Kinetics and Performance of Layered Composite Cathode Material Li [Li0. $2 \mathrm{Ni0}$. 2Mn0. 6] O2. J. Electrochem. Soc. 160, A2212-A2219 (2013).

102. Konishi, H., Gunji, A., Feng, X. \& Furutsuki, S. Effect of transition metal composition on electrochemical performance of nickel-manganese-based lithium-rich layer-structured cathode materials in lithium-ion batteries. J. Solid State Chem. 249, 80-86 (2017).

103. Noh, H.-J., Youn, S., Yoon, C. S. \& Sun, Y.-K. Comparison of the structural and electrochemical properties of layered $\mathrm{Li}[\mathrm{NixCoyMnz}] \mathrm{O} 2(\mathrm{x}=1 / 3,0.5,0.6,0.7,0.8$ and 0.85$)$ cathode material for lithium-ion batteries. $J$. Power Sources 233, 121-130 (2013).

104. Zheng, J., Kan, W. H. \& Manthiram, A. Role of Mn Content on the Electrochemical Properties of Nickel-Rich Layered LiNi0.8-xCo0.1Mn0.1+xO2 $(0.0 \leq \mathrm{x} \leq 0.08)$ Cathodes for Lithium-Ion Batteries. ACS Appl. Mater. Interfaces 7, 6926-6934 (2015).

105. Woo, S.-U. et al. Significant Improvement of Electrochemical Performance of AlF3-Coated Li [ Ni0.8Co0.1Mn0.1 ] O2 Cathode Materials. J. Electrochem. Soc. 154, A1005-A1009 (2007).

106. Lin, F. et al. Synchrotron X-ray Analytical Techniques for Studying Materials Electrochemistry in Rechargeable Batteries. Chem. Rev. (2017). doi:10.1021/acs.chemrev.7b00007

107. Li, Q. et al. Quantitative probe of the transition metal redox in battery electrodes through soft x-ray absorption spectroscopy. J. Phys. Appl. Phys. 49, 413003 (2016). 
$+$

a

2-D layered oxide
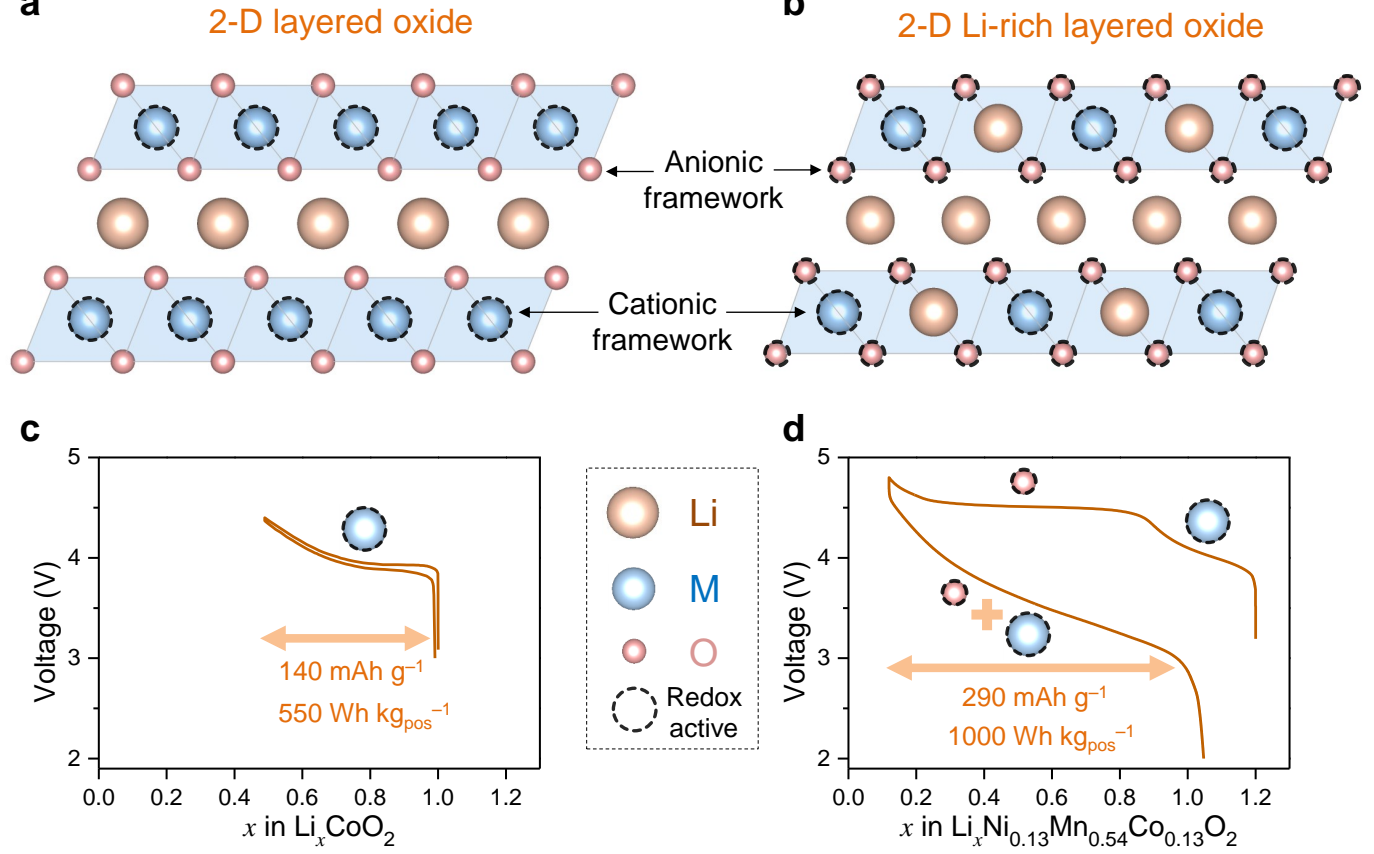

b

2-D Li-rich layered oxide

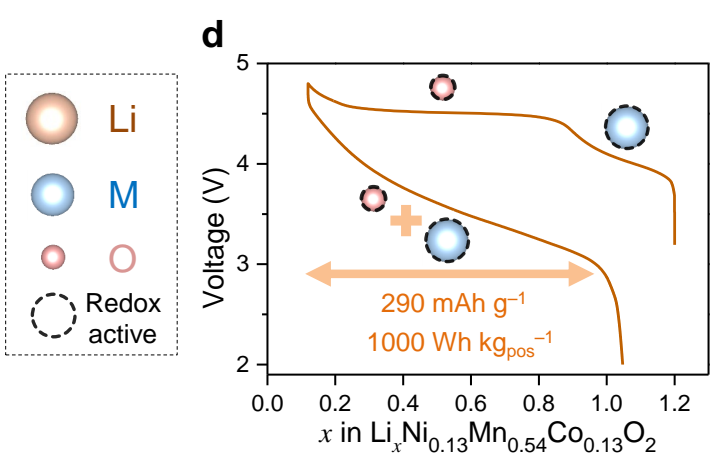


From Molecular Orbitals to the Band Structure

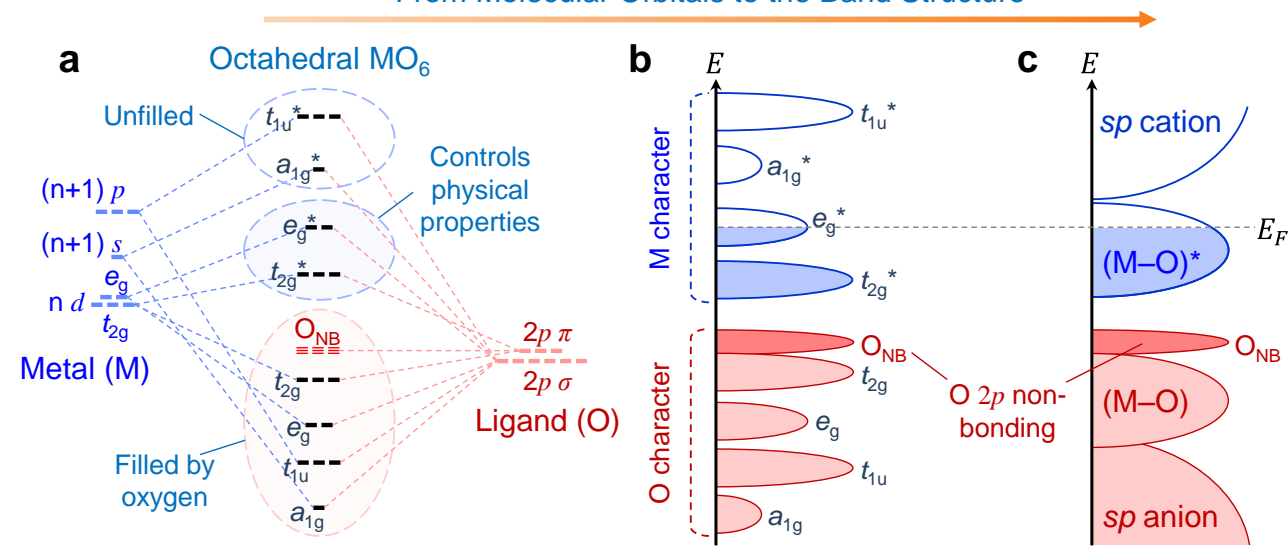

d $\mathrm{MO}_{2}$ slab in layered $\mathrm{LiMO}_{2}$
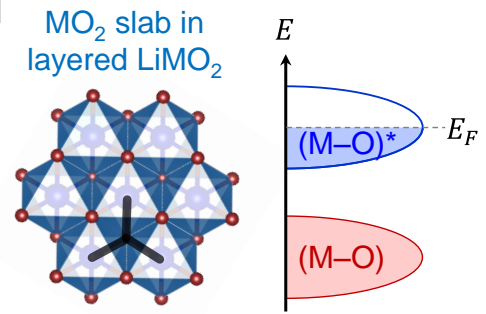

e
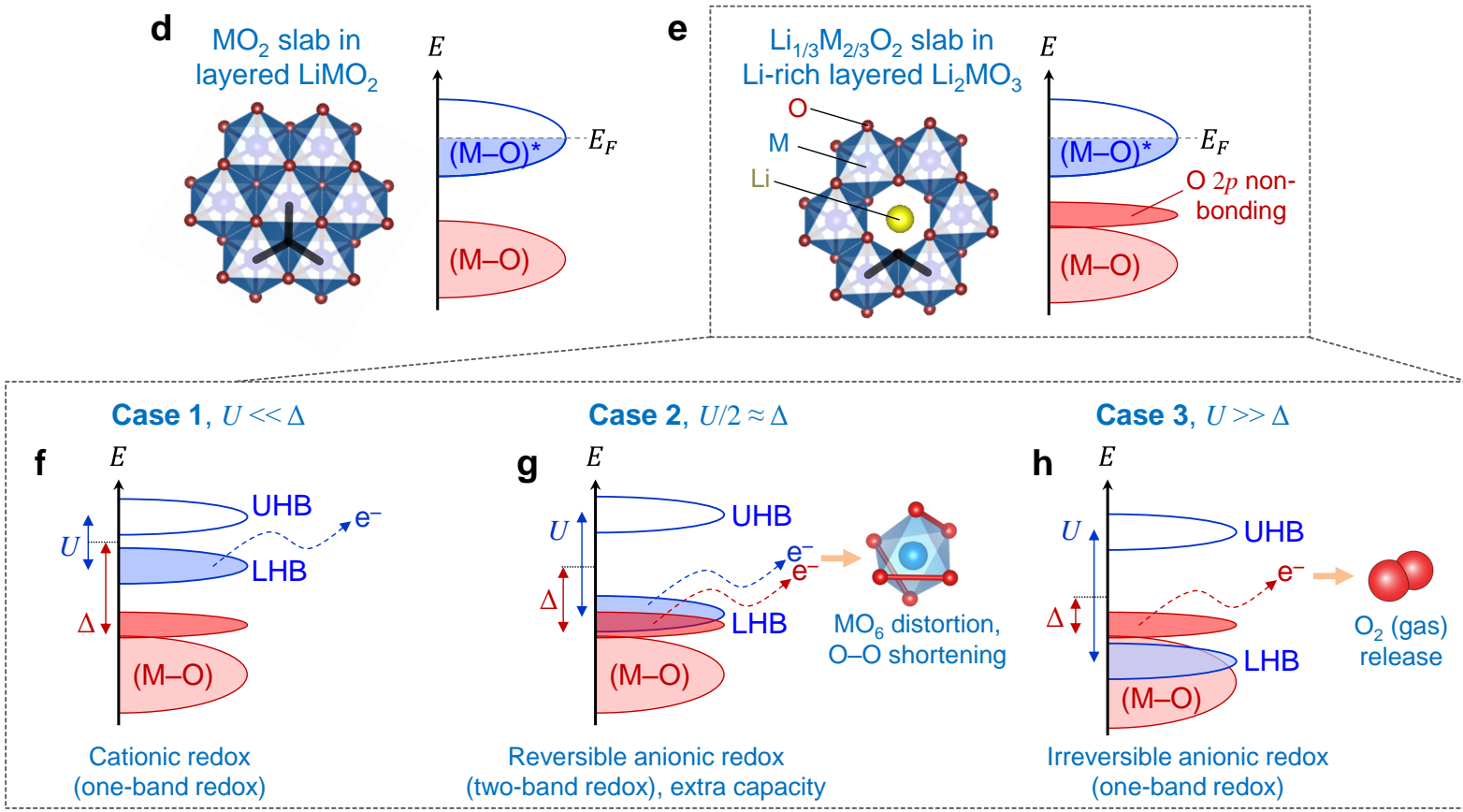


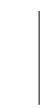

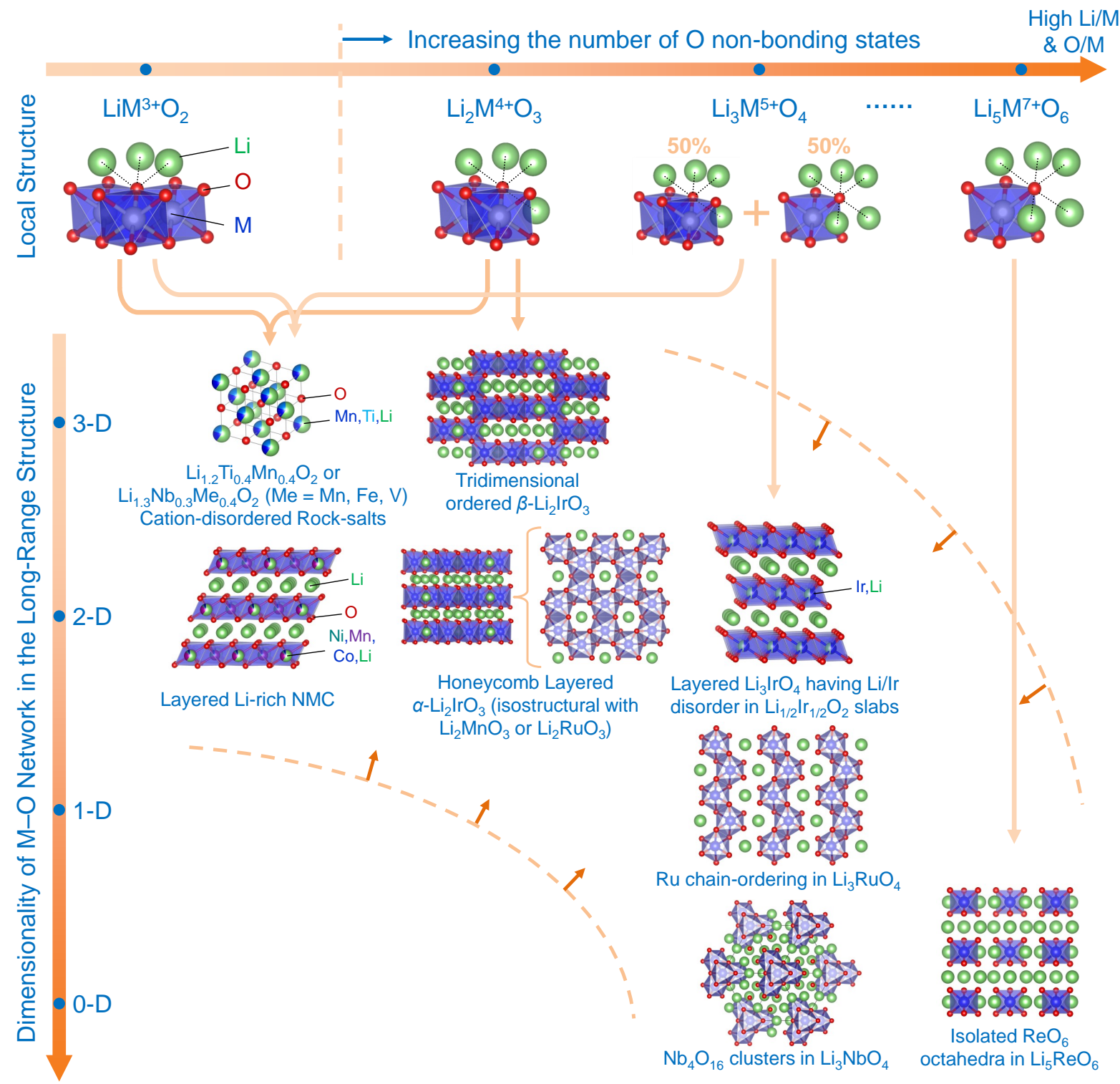



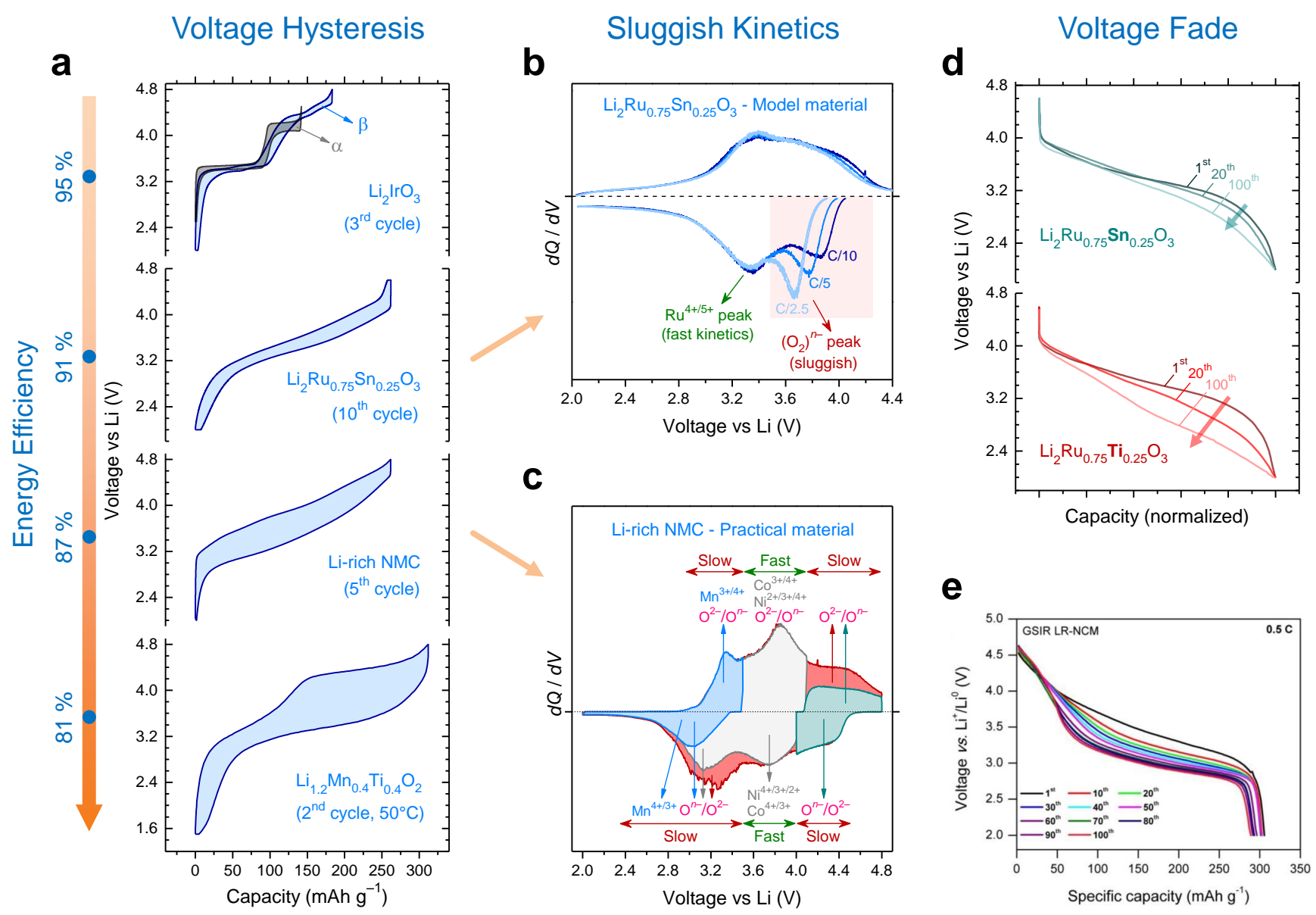

C
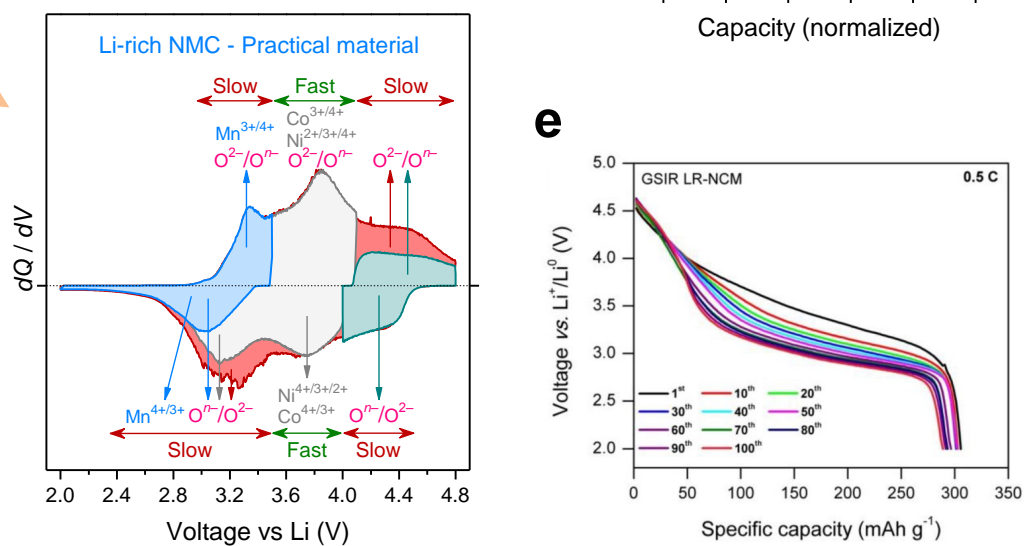
b

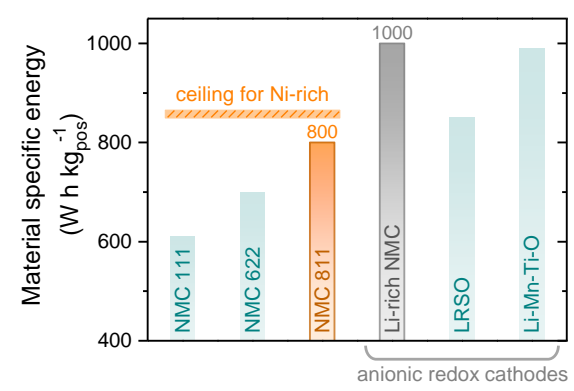

C

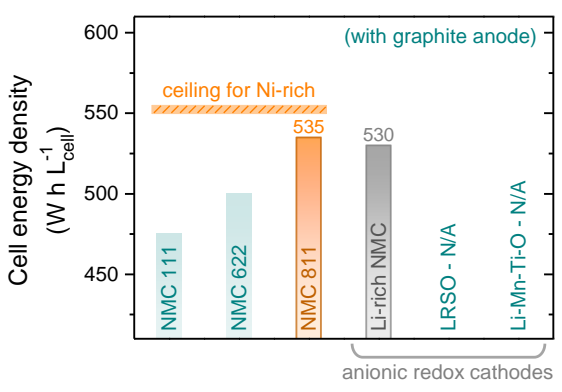

d

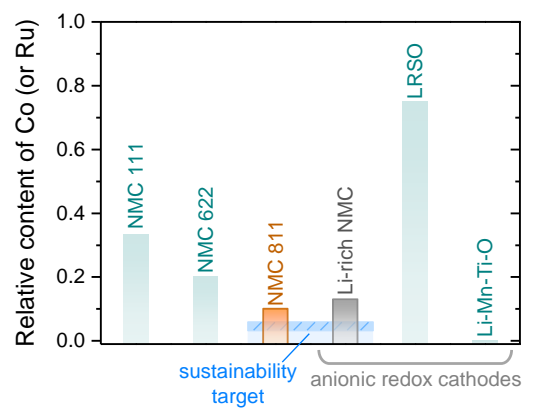

a $\square$ NMC 811

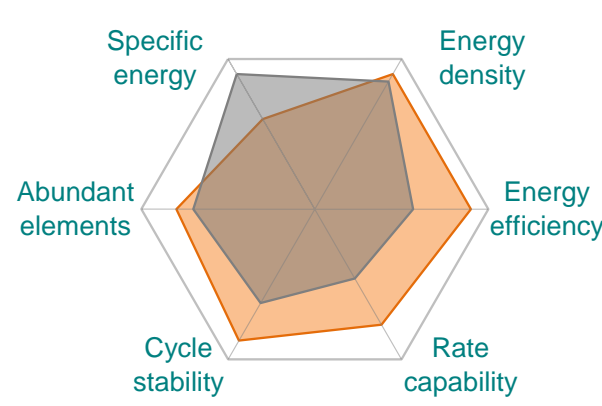

e

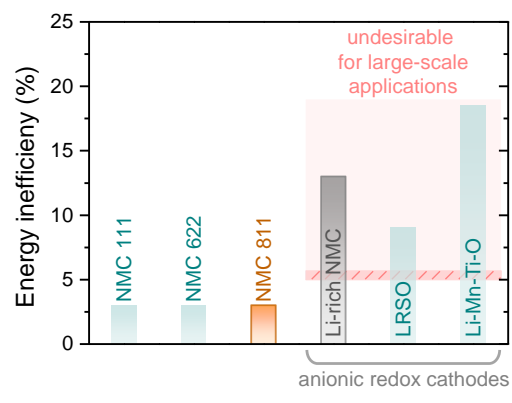

f

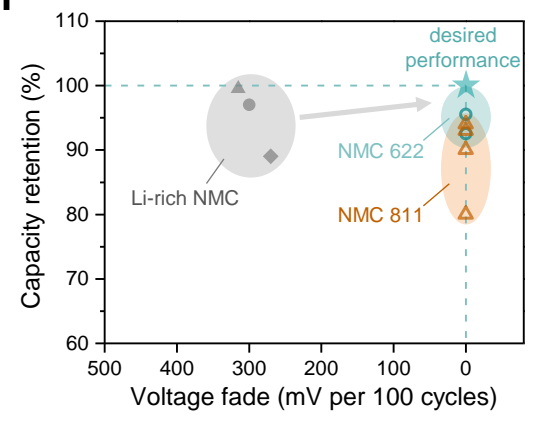

g

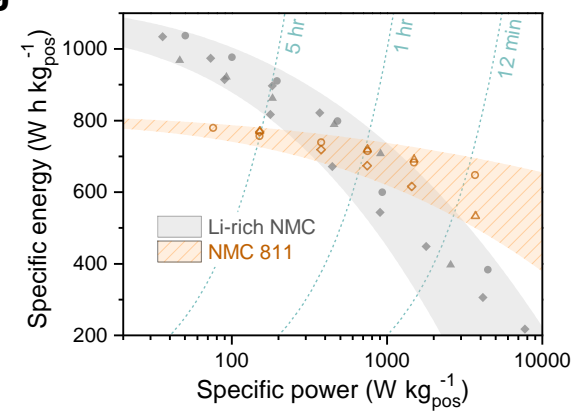



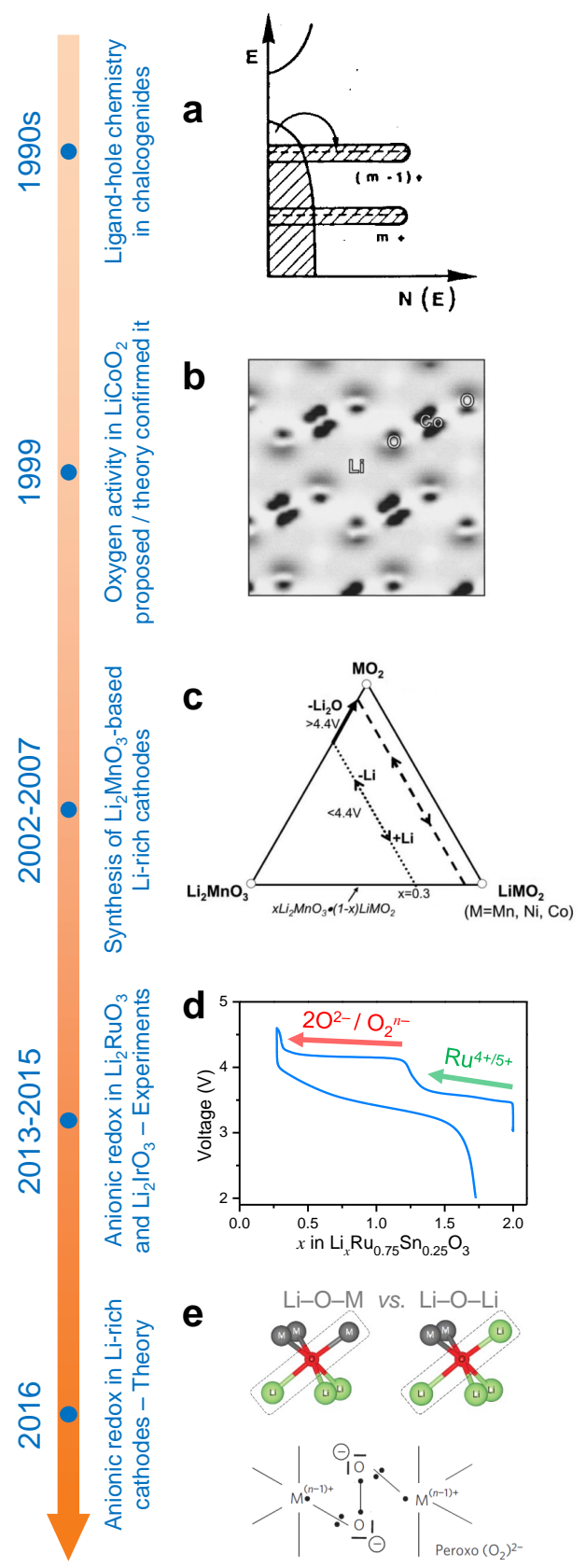

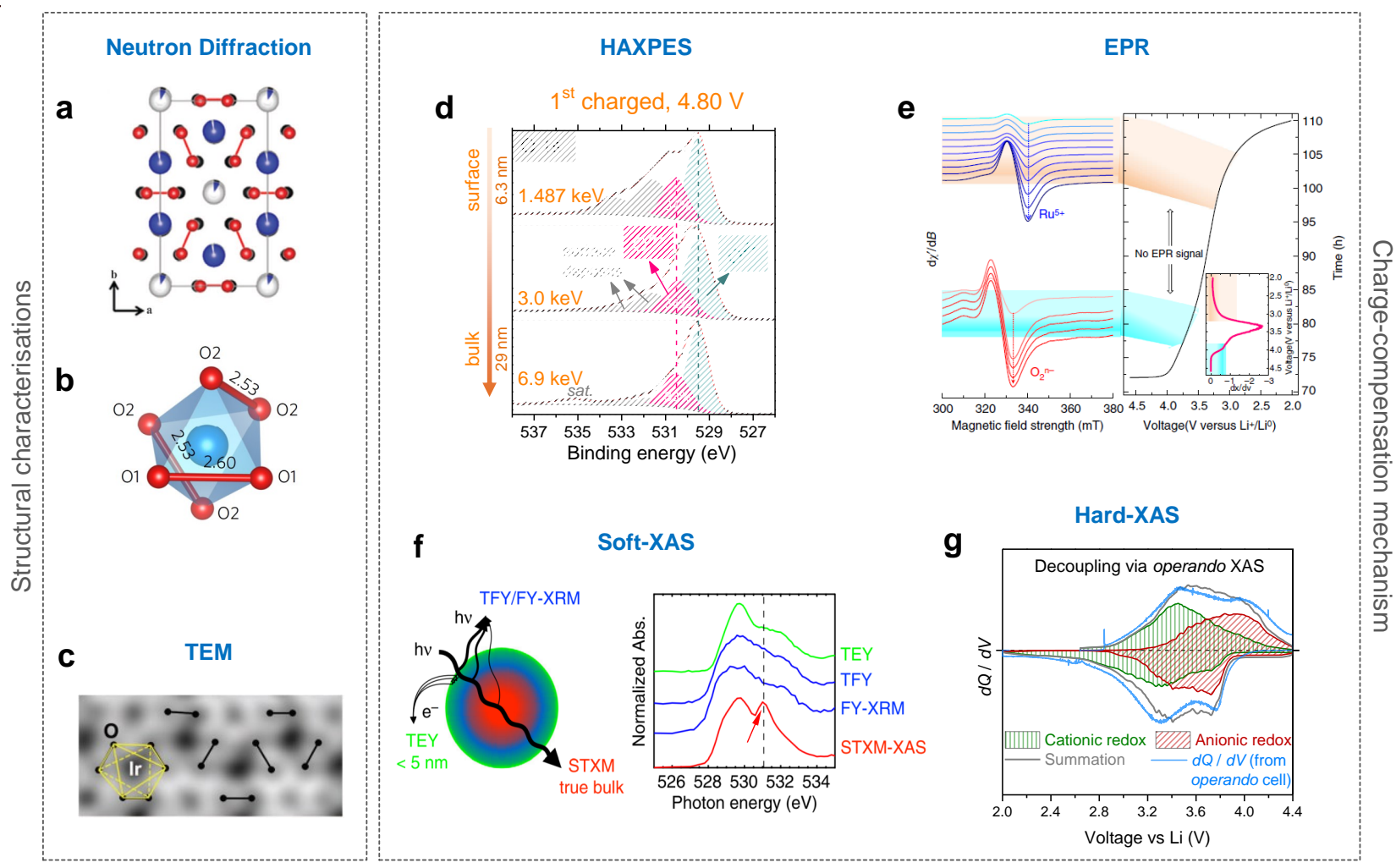